\title{
Runge-Kutta Type Integration Formulas Including the Evaluation of the Second Derivative Part I
}

By

Taketomo Mitsui*

\section{Introduction}

We are concerning with theoretical study of numerical integration procedure for the initial-value problem of ordinary differential equation:

$$
\begin{aligned}
& \frac{d y}{d x}=f(x, y), \\
& y\left(x_{I}\right)=y_{I} .
\end{aligned}
$$

Many numerical analysts have been investigating the discrete variable methods for the problem. Consequently everyone can enjoy to solve numerically ordinary differential equation in almost all computing centers. It seems as if we got the numerical integrator through the use of the computer. But the study is yet continued for "better" numerical procedure.

Among the one-step methods, Runge-Kutta methods (RK methods, in short) are popular because of the high accuracy and the feasibility of changing step-size. In general the methods are expressed as follows. The solution of (E) at $x_{0}+h$ is approximated by

$$
y_{1}=y_{0}+h \sum_{i=1}^{p} \mu_{i} k_{i}
$$

where

(*)

$$
\begin{aligned}
& k_{1}=f\left(x_{0}, y_{0}\right), \\
& k_{i}=f\left(x_{0}+\alpha_{i} h, y_{0}+h \sum_{j=1}^{i-1} \beta_{i j} k_{j}\right), \quad i=2, \ldots, p,
\end{aligned}
$$

Received February 27, 1981.

* Research Institute for Mathematical Sciences, Kyoto University, Kyoto 606, Japan. 
$h$ is the step-size and $y_{0}$ is the approximated value at $x_{0}$. This type of the method is called (explicit) $p$-stage Runge-Kutta algorithm. According to the choice of the stage number $p$ and the parameters $\alpha_{i}, \beta_{i j}, \mu_{i}$ we have many variations, among which the classical Runge-Kutta method or the Runge-Kutta-Fehlberg method is famous. A distinguished contribution for the study of the RungeKutta methods has been made by J. C. Butcher ([1] [4]). He determined the attainable order of the RK methods up to 10-stage formula. On the other hand he introduced the semi-explicit (the summation is up to $i$ instead of $i-1$ in $(*)$ ) or implicit (the summation is up to $p$ in $(*)$ ) formula.

RK methods (and perhaps many other quadrature formulas for the initialvalue problem) are constructed on the principle that the required function evaluation is only for $f(x, y)$, i.e. the first derivative of the solution. It is quite natural because we are acquainted with the functional form of the first derivative in the ordinary differential equation. Recently, however, some propositions have been made to employ the function evaluation of the second derivative of the solution. Functional form of it is given by

$$
g(x, y)=f_{x}(x, y)+f_{y}(x, y) f(x, y) .
$$

M. URABE [15] made a first attempt to employ $g(x, y)$ by presenting an implicit one-step method with step-size control strategy. Let $y_{0}$ and $y_{-1}$ be approximations of $y(x)$ at $x_{0}$ and $x_{0}-h$, respectively. His algorithm employs the predictor given by

$$
\hat{y}_{1}=-31 y_{-1}+32 y_{0}-h\left(14 f_{-1}+16 f_{0}\right)+h^{2}\left(-2 g_{-1}+4 g_{0}\right)
$$

and the corrector given by

$$
\begin{aligned}
y_{0}=y_{-1} & +\frac{h}{240}\left(101 f_{-1}+128 f_{0}+11 \hat{f}_{1}\right) \\
& +\frac{h^{2}}{240}\left(13 g_{-1}-40 g_{0}-3 \hat{g}_{1}\right),
\end{aligned}
$$

where $f_{i}=f\left(x_{0}+i h, y_{i}\right), g_{i}=f\left(x_{0}+i h, y_{i}\right), \hat{f}_{1}=f\left(x_{0}+h, \hat{y}_{1}\right)$ and $\hat{g}_{1}=g\left(x_{0}+h\right.$, $\hat{y}_{1}$ ). Succeeding his result, J. R. CASH [5] has considered this type of formula more generally and made some stability analysis. On the other hand H. SHINTANI [12], [13] has proposed some formulas analogous to RK formula employing one evaluation for $f(x, y)$ and some for $g(x, y)$. He has given the values of the parameters appearing in the formulas up to the order 7 . His results, closely related to the present work, will be mentioned afterward. 
In this context another type of integration formula, for the origination of which H. H. Rosenbrock [11] is given credit, is now being developed. It employs the partial derivative $f_{y}(x, y)$ and is reported to have good stability for stiff systems of ordinary differential equations ([7], [9]).

Here we shall examine an explicit $(p, q)$-stage Runge-Kutta type formula including the second derivative. It requires $p$ times evaluations for the first derivative and $q$ times for the second derivative in a similar manner for $R K$ methods. We are interested in the following problems.

(1) What is the attainable order of the $(p, q)$-stage formula from the viewpoint of its local accuracy?

(2) How are the parameters in the formula determined?

(3) What formula is good for practical use?

These problems will be solved in the following sections and the forthcoming paper by the author. The present paper is especially devoted to investigate the $(1, q)$-stage formulas.

First, we shall define explicit $(p, q)$-stage formula. Next, some algebraic computations are carried out to investigate $(p, q)$-stage formulas. Here SAM software is used as a powerful tool. Then, the attainable order of $(1, q)$-stage formula is determined up to $q=4$.

Remark. In the case of very complicated functional form of $f(x, y)$ in higher dimension, the calculation of the second derivative $g(x, y)$ requires a laborious work. It is the main reason why the methods employing $g(x, y)$ have not been considered. But the recent development of the symbolic and algebraic manipulation (SAM) software brings the change of the situation. SAM software, for example, REDUCE-2 or MACSYMA, is now a helpful tool for mathematical sciences. In fact, some SAM program may print expressions in a FORTRAN notation so that one can carry out the calculation of the second derivative from $f(x, y)$ in an automatic way. Once after algebraic computation we may call $g(x, y)$ as a FUNCTION subprogram.

Moreover, SAM software is very useful for the theoretical study of the $\mathbb{R K}$ and its analogous methods. For example, H. TodA [14] has considered 5-stage RK limiting formula of order 5. He has utilized MACSYMA essentially. We shall also attempt to apply SAM for our study. 


\section{Acknowledgement}

The present author would like to express sincere thanks to Prof. S. Hirotumatu for his constant encouragement and helpful discussions during the preparation of the work. $\mathrm{He}$ is also greatly indebted to Prof. K. ICHIDA for carrying out the computations by interval arithmetic.

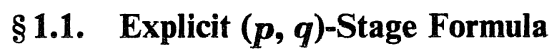

We shall discuss numerical integration procedure for the initial-value problem of ordinary differential equation:

$$
\begin{aligned}
& \frac{d y}{d x}=f(x, y), \\
& y\left(x_{I}\right)=y_{I} .
\end{aligned}
$$

Here $f$ is sufficiently smooth with respect to $x$ and $y$. Let us define an explicit $(p, q)$-stage Runge-Kutta type formula including the second derivative of the solution. Let $g$ stand for the second derivative of $y(x)$,

$$
g(x, y)=f_{x}(x, y)+f_{y}(x, y) f(x, y) .
$$

Explicit $(p, q)$-stage formula is given as follows.

$$
y_{n+1}=y_{n}+h \sum_{i=1}^{p} \mu_{i} k_{i}+h^{2} \sum_{i=1}^{q} v_{i} K_{i}, \quad n=0,1,2 \ldots
$$

where

$$
\begin{aligned}
& \left\{\begin{array}{l}
k_{1}=f\left(x_{n}+\alpha_{1} h, y_{n}\right), \\
k_{i}=f\left(x_{n}+\alpha_{i} h, y_{n}+h \sum_{j=1}^{i-1} \beta_{i j} k_{j}+h^{2} \sum_{j=1}^{i-1} \gamma_{i j} K_{j}\right), \quad i=2, \ldots, p,
\end{array}\right. \\
& \left\{\begin{array}{l}
K_{1}=g\left(x_{n}+\rho_{1} h, y_{n}+h \sigma_{11} k_{1}\right), \\
K_{i}=g\left(x_{n}+\rho_{i} h, y_{n}+h \sum_{j=1}^{i} \sigma_{i j} k_{j}+h^{2} \sum_{j=1}^{i-1} \tau_{i j} K_{j}\right), \quad i=2, \ldots, q .
\end{array}\right.
\end{aligned}
$$

Remark 1. The parameters $\mu_{i}, v_{i}, \alpha_{i}, \beta_{i j}, \gamma_{i j}, \rho_{i}, \sigma_{i j}$ and $\tau_{i j}$ are, of course, real numbers.

Remark 2. In the case of simultaneous equations in (1), $y$ and $f$ are considered vectors of the same dimension. Then $f_{y}(x, y)$, the Jacobian matrix of $f$, is given in the matrix form. For example, assume that 


$$
f(x, y)=\left(\begin{array}{l}
f_{1}\left(y_{1}, y_{2}\right) \\
f_{2}\left(y_{1}, y_{2}\right)
\end{array}\right)=\left(\begin{array}{c}
\left(a_{1}-b_{1} y_{2}\right) y_{1} \\
\left(a_{2}-b_{2} y_{1}\right) y_{2}
\end{array}\right)
$$

where $a_{i}$ and $b_{i}$ are constants, then

$$
f_{\mathrm{r}}(x, y)=\left(\begin{array}{cc}
a_{1}-b_{1} y_{2} & -b_{1} y_{1} \\
-b_{2} y_{2}^{\prime} & a_{2}-b_{2} y_{1}
\end{array}\right)
$$

and

$$
g(x, y)=\left(\begin{array}{l}
a_{1}^{2} y_{1}+\left(b_{2} y_{1}+b_{1} y_{2}-2 a_{1}-a_{2}\right) b_{1} y_{1} y_{2} \\
a_{2}^{2} y_{2}+\left(b_{2} y_{1}+b_{1} y_{2}-a_{1}-2 a_{2}\right) b_{2} y_{1} y_{2}
\end{array}\right) .
$$

Similar to the RK formulas, the detcrmining equations and the parameters are possible to be slightly different between the single differential equation and the systems of equations. For convenience sake we shall investigate the single case. The attainable order of the formula is not depend on whether (1) is single or system.

\section{$\S 1.2$. The Taylor Series Expansion of the Solution}

To investigate Runge-Kutta type methods of higher order, we are required to represent the solution $y\left(x_{0}+h\right)$ for (E) and (IV) in Section 1.1 into the power series of the stepsize $h$. For our quadrature formula it is preferable to represent the solution into the power series utilizing the second derivative $g$.

Twice integration after differentiation for the equation

$$
\frac{d y}{d x}=f(x, y), \quad x \geqq x_{0}
$$

implies the formula

$$
\int_{x_{0}}^{x_{0}+h}\left\{\int_{x_{0}}^{t} \frac{d^{2} y(x)}{d x^{2}} d x\right\} d t=\int_{x_{0}}^{x_{0}+h}\left\{\int_{x_{0}}^{t} g(x, y(x)) d x\right\} d t
$$

The left-hand side of the above formula is equal to

$$
\begin{aligned}
& y\left(x_{0}+h\right)-y\left(x_{0}\right)-h f\left(x_{0}, y\left(x_{0}\right)\right) \\
& \quad=y\left(x_{0}+h\right)-y_{0}-h f_{0} .
\end{aligned}
$$

Hereafter the subscript 0 stands for the evaluation at $x=x_{0}$ and $y=y_{0}$.

The right-hand side of (1) becomes

$$
\int_{0}^{h}\left\{\int_{x_{0}}^{x_{0}+\xi} g(x, y(x)) d x\right\} d \xi=\int_{0}^{h}\left\{\int_{0}^{\xi} g\left(x_{0}+\zeta, y\left(x_{0}+\zeta\right)\right) d \zeta\right\} d \zeta
$$


Then, assume that for $0 \leqq \zeta \leqq h, y\left(x_{0}+\zeta\right)$ can be expanded in the power series of $\zeta$ by

$$
y\left(x_{0}+\zeta\right)=y_{0}+\zeta f_{0}+\sum_{r=2}^{m} \frac{\kappa_{r-2}}{r !} \zeta^{r}+O\left(\zeta^{m+1}\right),
$$

where $\kappa_{j}(j=1,2, \ldots, m)$ is the coefficient to be determined later. Substitution (2) into the integrand implies

$$
\begin{aligned}
g\left(x_{0}+\zeta, y\left(x_{0}+\zeta\right)\right)= & g\left(x_{0}+\zeta, y_{0}+\zeta f_{0}+\sum_{r=0}^{m} \frac{\kappa_{r-2}}{r !} \zeta^{r}+O\left(\zeta^{m+1}\right)\right) \\
= & \sum_{l=0}^{m} \frac{1}{l !}\left[\left(\zeta \frac{\partial}{\partial x}+\left(\zeta f_{0}+\sum_{r=0}^{m} \frac{\kappa_{r-2}}{r !} \zeta^{r}\right) \frac{\partial}{\partial y}\right)^{l} g\right]_{0}+O\left(\zeta^{m+1}\right) \\
= & \sum_{l=0}^{m} \frac{\zeta^{l}}{l !}\left[\left(D_{0}+\left(\sum_{r=2}^{m} \frac{\kappa_{r-2}}{r !} \zeta^{r-1}\right) \frac{\partial}{\partial y}\right)^{l} g\right]_{0}+O\left(\zeta^{m+1}\right) \\
= & \sum_{l=0}^{m} \frac{\zeta^{l}}{l !}\left\{\sum_{k=0}^{l}\left(\begin{array}{l}
l \\
k
\end{array}\right)\left(\sum_{r=1}^{m-1} \frac{\kappa_{r-1}}{(r+1) !} \zeta^{r}\right)^{k}\right. \\
& \left.\times\left[D_{0}^{l-k}\left(\frac{\partial}{\partial y}\right)^{k} g\right]_{0}\right\}+O\left(\zeta^{m+1}\right) .
\end{aligned}
$$

Here $D_{0}$ is a differential operator defined by

$$
D_{0}=\frac{\partial}{\partial x}+f_{0} \frac{\partial}{\partial y} .
$$

Therefore we have the following equation:

$$
\begin{aligned}
y_{0} & +h f_{0}+\sum_{r=2}^{m+2} \frac{\kappa_{r-2}}{r !} \zeta^{r}=y_{0}+h f_{0}+ \\
& +\int_{0}^{h}\left(\int_{0}^{\xi} \sum_{l=0}^{m} \frac{\zeta^{l}}{l !}\left\{\sum_{k=0}^{l}\left(\begin{array}{l}
l \\
k
\end{array}\right)\left(\sum_{r=1}^{m-1} \frac{\kappa_{r-1}}{(r+1) !} \zeta^{r}\right)^{k}\left[D_{0}^{l-k}\left(\frac{\partial}{\partial y}\right) g\right]_{0}\right\} d \zeta\right) d \xi,
\end{aligned}
$$

which determines $\kappa_{j}$ recurrently.

Twice differentiation for (3) with respect to $h$ implies

$$
\sum_{l=0}^{m} \frac{\kappa_{l}}{l !} h^{l}=\sum_{l=0}^{m} \frac{h^{l}}{l !}\left\{\sum_{k=0}^{l}\left(\begin{array}{l}
l \\
k
\end{array}\right)\left(\sum_{r=1}^{m-1} \frac{\kappa_{r-1}}{(r+1) !} h^{r}\right)^{k}\left[D_{0}^{l-k}\left(\frac{\partial}{\partial y}\right)^{k} g\right]_{0}\right\} .
$$

Then, we have the following important result.

Theorem 1. The coefficient $\kappa_{l}(l=0,1, \ldots, m)$ is determined by the following way:

$$
\left\{\begin{array}{l}
\kappa_{0}=g_{0} . \\
\kappa_{l}=\left[D_{0}^{l} g\right]_{0} \\
+\sum_{s=1}^{l-1}\left\{\sum_{t=1}^{\mathrm{m}(l, s)} \frac{l !}{s !(l-s-t) !}\right. \\
\left.\times B_{s, t}\left(\frac{\kappa_{0}}{2}, \frac{\kappa_{1}}{3}, \ldots, \frac{\kappa_{s-1}}{s+1}\right)\left[D_{0}^{l-s-t}\left(\frac{\partial}{\partial y}\right)^{t} g\right]_{0}\right\} \\
\text { for } l=1,2, \ldots, m
\end{array}\right.
$$


Here $B_{s, t}\left(x_{1}, x_{2}, \ldots, x_{s}\right)$ is the multivariable polynomial of E. T. Bell of the order $(s, t)$ and

$$
\mathrm{m}(l, s) \equiv \min (s, l-s) .
$$

Note. $\quad \kappa_{j}$ appeared in the summation of the right-hand side of (5) is for $j$ less than or equal to $l-2$ so that we can determine $\kappa_{l}$ recurrently by the formula.

Proof. $\quad \kappa_{0}=g_{0}$ is clear. Let us introduce two functions $G(h)$ and $A(h ; l)$ as follows:

$$
\begin{aligned}
& G(h)=\sum_{r=1}^{m-1} \frac{\kappa_{r-1}}{(r+1) !} h^{r}, \\
& A(h ; l)=\sum_{k=0}^{l}\left(\begin{array}{l}
l \\
k
\end{array}\right)\{G(h)\}^{k}\left[D_{0}^{l-k}\left(\frac{\partial}{\partial y}\right)^{k} g\right]_{0}, l=0,1, \ldots, m .
\end{aligned}
$$

Note that $G(h)$ is of the order $h$ and $G(0)=0$ holds. Then, the right-hand side of (4) is equal to $\sum_{s=0}^{m} \frac{A(h ; s)}{s !} h^{s}$.

Hence, we have the equation

$$
\sum_{l=0}^{m} \frac{\kappa_{l}}{l !} h^{l}=\sum_{s=0}^{m} \frac{A(h ; s)}{s !} h^{s} .
$$

For an integer $l$ such that $1 \leqq l \leqq m, l$-times differentiation to (8) with respect to $h$ implies

$$
\kappa_{l}=\sum_{s=0}^{l-1}\left(\begin{array}{l}
l \\
s
\end{array}\right) A^{(s)}(0 ; l-s) .
$$

By (7), the equation

$$
A(0 ; l)=\left[D_{0}^{l} g\right]_{0}
$$

is clear. For $s \geqq 1$, we have from (7)

$$
\begin{aligned}
A^{(s)}(h ; l-s)= & \left(\begin{array}{c}
l-s \\
1
\end{array}\right) G^{(s)}(h)\left[D_{0}^{l-s-1} g_{y}\right]_{0} \\
& +\left(\begin{array}{c}
l-s \\
2
\end{array}\right) \frac{d^{s}}{d h^{s}}\{G(h)\}^{2}\left[D_{0}^{l-s-2} g_{y y}\right]_{0}+\cdots \\
& +\left(\begin{array}{c}
l-s \\
k
\end{array}\right) \frac{d^{s}}{d h^{s}}\{G(h)\}^{k}\left[D_{0}^{l-s-k}\left(\frac{\partial}{\partial y}\right)^{k} g\right]_{0}+\cdots \\
& +\frac{d^{s}}{d h^{s}}\{G(h)\}^{l-s}\left[\left(\frac{\partial}{\partial y}\right)^{l-s} g\right]_{0} .
\end{aligned}
$$


For higher derivatives of composite functions, the formula employing the Bell polynomial is known (J. RIORDAN [10]). Hence, applying the formula, we have for $k=1,2, \ldots, l-s$

$$
\frac{d^{s}}{d h^{s}}\{G(h)\}^{k}=\sum_{t=1}^{\min (s, k)} \frac{k !}{(k-t) !} B_{s, t}\left(G^{\prime}(h), G^{\prime \prime}(h), \ldots, G^{(s)}(h)\right) \times\{G(h)\}^{k-t},
$$

which implies

$$
\left[\frac{d^{s}}{d h^{s}}\{G(h)\}^{k}\right]_{h=0}= \begin{cases}k ! B_{s, k}\left(G^{\prime}(0), G^{\prime \prime}(0), \ldots, G^{(s)}(0)\right) & \text { for } s \geqq k \\ 0 & \text { for } s<k\end{cases}
$$

From (6), the equation

$$
G^{(s)}(h)=\sum_{r=s}^{m-1} \frac{r !}{(r-s) !} \frac{\kappa_{r-1}}{(r+1) !} h^{r-s}=\sum_{r=s}^{m-1} \frac{\kappa_{r-1}}{(r+1) !(r-s) !} h^{r-s}
$$

holds. Hence we have

$$
G^{(s)}(0)=\frac{\kappa_{s-1}}{s+1}, \quad s=1,2, \ldots, m-1
$$

Then $A^{(s)}(0 ; l-s)$ is given by

$$
\begin{aligned}
A^{(s)}(0, l-s) & =\sum_{k=1}^{\mathrm{m}(l, s)}\left(\begin{array}{c}
l-s \\
k
\end{array}\right) k ! B_{s, t}\left(G^{\prime}(0), \ldots, G^{(s)}(0)\right)\left[D_{0}^{l-s-k}\left(\frac{\partial}{\partial y}\right)^{k} g\right]_{0} \\
& =\sum_{t=1}^{\mathrm{m}(l, s)} \frac{(l-s) !}{(l-s-t) !} B_{s, t}\left(\frac{\kappa_{0}}{2}, \frac{\kappa_{1}}{3}, \ldots, \frac{\kappa_{s-1}}{s+1}\right)\left[D_{0}^{l-s-t}\left(\frac{\partial}{\partial y}\right)^{t} g\right]_{0},
\end{aligned}
$$

which implies from (9)

$$
\begin{aligned}
& \kappa_{l}=\left[D_{0}^{l} g\right]_{0}+\sum_{s=1}^{l-1}\left(\begin{array}{c}
l \\
s
\end{array}\right)\left\{\sum_{t=1}^{\mathrm{m}(l, s)} \frac{(l-s) !}{(l-s-t) !} B_{s, t}\left(\frac{\kappa_{0}}{2}, \frac{\kappa_{1}}{3}, \ldots, \frac{\kappa_{s-1}}{s+1}\right)\right. \\
&\left.\times\left[D_{0}^{l-s-t}\left(\frac{\partial}{\partial y}\right)^{t} g\right]_{0}\right\} \\
&=\left[D_{0}^{l} g\right]_{0}+\sum_{s=1}^{l-1}\left\{\sum _ { t = 1 } ^ { \mathrm { m } ( l , s ) } \frac { l ! } { s ! ( l - s - t ) ! } B _ { s , t } \left(\frac{\kappa_{0}}{2},\right.\right.\left.\frac{\kappa_{1}}{3}, \ldots, \frac{\kappa_{s-1}}{s+1}\right) \\
&\left.\times\left[D_{0}^{l-s-t}\left(\frac{\partial}{\partial y}\right)^{t} g\right]_{0}\right\} .
\end{aligned}
$$

This is the desired result.

The multivariable polynomial $B_{s, t}\left(x_{1}, x_{2}, \ldots, x_{s}\right)$ has a recurrence formula to calculate it conveniently by the application of any SAM software. That is, 


$$
\begin{aligned}
\sum_{t=1}^{s} & x_{1} B_{s, t}\left(x_{1}, \ldots, x_{s}\right) a^{t+1}+\sum_{t=1}^{s}\left(\sum_{k=1}^{s} x_{k+1} \frac{\partial B_{s, t}}{\partial x_{k}}\right) a^{t} \\
= & \sum_{t=1}^{s+1} B_{s+1, t}\left(x_{1}, \ldots, x_{s+1}\right) a^{l}
\end{aligned}
$$

Here, the both sides of the formula is considered as a polynomial of $a$. The recurrence formula for $B_{s, t}$ will be employed during the calculations of $\kappa_{l}$ by REDUCE-2.

Theorem 1 tells us a concrete method to determine the Taylor series expansion (2) employing the second derivative. For example,

$$
\begin{aligned}
\kappa_{1} & =\left[D_{0} g\right]_{0}, \\
\kappa_{2} & =\left[D_{0}^{2} g\right]_{0}+2 ! B_{1,1}\left(\frac{\kappa_{0}}{2}\right) g_{y, 0}=\left[D_{0}^{2} g\right]_{0}+\kappa_{0} g_{y, 0}=\left[D_{0}^{2} g\right]_{0}+g_{0} g_{y, 0}, \\
\kappa_{3} & =\left[D_{0}^{3} g\right]_{0}+3 ! B_{1,1}\left(\frac{\kappa_{0}}{2}\right)\left[D_{0} g_{y}\right]_{0}+\frac{3 !}{2 !} B_{2,1}\left(\frac{\kappa_{0}}{2}, \frac{\kappa_{1}}{3}\right) g_{y, 0} \\
& =\left[D_{0}^{3} g\right]_{0}+3 \kappa_{0}\left[D_{0} g_{y}\right]_{0}+\kappa_{1} g_{y, 0} \\
& =\left[D_{0}^{3} g\right]_{0}+3 g_{0}\left[D_{0} g_{y}\right]_{0}+\left[D_{0} g\right]_{0} g_{y, 0} .
\end{aligned}
$$

We may also carry out the process by the SAM software up to the desired order. The result by REDUCE-2 is shown in Table 1.

Note that because of Remark 2 in the preceding section we do not care the order of the higher partial derivatives.

Another important result from Theorem 1 is that any $\kappa_{j}$ is a summation of an integer multiple of some product of $\left[D_{0}^{p}(\partial / \partial y)^{q} g\right]_{0}$. Hence, we shall call any product of $\left[D_{0}^{p}(\partial / \partial y)^{q} g\right]_{0}$ an elementary differential of $g$. J. C. ButcheR [1] has used the terminology of elementary differential in a different sense. However, our study of the expression of the coefficient of Taylor series in the elementary differentials is on the similar point of view.

In fact, the way of the proof of Theorem 1 is applicable to the Taylor series expansion of $y\left(x_{0}+h\right)$ employing the first derivative.

\section{Theorem 2. Assume that}

$$
y\left(x_{0}+h\right)=y_{0}+\sum_{r=1}^{m} \frac{\lambda_{r-1}}{r !} h^{r}+O\left(h^{m+1}\right) .
$$

Then, the coefficient $\lambda_{l}(l=0,1, \ldots, m-1)$ is determined by 


$$
\left\{\begin{aligned}
& \lambda^{0}= f_{0} \\
& \lambda_{l}= {\left[D_{0}^{l} f\right]_{0}+\sum_{s=1}^{l-1}\left\{\sum_{t=1}^{\mathrm{m}(l, s)} \frac{l !}{(l-s-t) ! s !}\right.} \\
&\left.\times B_{s, t}\left(\frac{\lambda_{1}}{2}, \frac{\lambda_{2}}{3}, \ldots, \frac{\lambda_{s}}{s+1}\right)\left[D_{0}^{l-s-t}\left(\frac{\partial}{\partial y}\right)^{t} f\right]_{0}\right\}, \\
& l=1,2, \ldots, m .
\end{aligned}\right.
$$

We shall call (10) the first type power series expansion in contrast with (2) which will be mentioned as the second type expansion.

Table 1.

$$
\begin{aligned}
& \kappa_{0}=g_{0} \\
& \kappa_{1}=\left[D_{0} g\right]_{0} \\
& \kappa_{2}=\left[D_{0}^{2} g\right]_{0}+g_{0} \cdot g_{y, 0} \\
& \kappa_{3}=\left[D_{0}^{3} g\right]_{0}+\left[D_{0} g\right]_{0} \cdot g_{y, 0}+3 g_{0} \cdot\left[D_{0} g_{y}\right]_{0} \\
& \kappa_{4}=\left[D_{0}^{4} g\right]_{0}+\left[D_{0}^{2} g\right]_{0} \cdot g_{y, 0}+4\left[D_{0} g\right]_{0} \cdot\left[D_{0} g_{y}\right]_{0}+6 g_{0} \cdot\left[D_{0}^{2} g_{y}\right]_{0}+g_{0} \cdot g_{y, 0}^{2} \\
& +3 g_{0}^{2} \cdot g_{y y, 0} \\
& \kappa_{5}=\left[D_{0}^{5} g\right]_{0}+\left[D_{0}^{3} g\right]_{0} \cdot g_{y, 0}+5\left[D_{0}^{2} g\right]_{0} \cdot\left[D_{0} g_{y}\right]_{0}+10\left[D_{0} g\right]_{0} \cdot\left[D_{0}^{2} g_{y}\right]_{0} \\
& +10 g_{0} \cdot\left[D_{0}^{3} g_{y}\right]_{0}+\left[D_{0} g\right]_{0} \cdot g_{y, 0}^{2}+8 g_{0} \cdot g_{y, 0} \cdot\left[D_{0} g_{y}\right]_{0}+10 g_{0} \cdot\left[D_{0} g\right]_{0} \cdot g_{y y, 0} \\
& +15 g_{0}^{2} \cdot\left[D_{0} g_{y y}\right]_{0} \\
& \kappa_{6}=\left[D_{0}^{6} g\right]_{0}+\left[D_{0}^{4} g\right]_{0} \cdot g_{y, 0}+6\left[D_{0}^{3} g\right]_{0} \cdot\left[D_{0} g_{y}\right]_{0}+15\left[D_{0}^{2} g\right]_{0} \cdot\left[D_{0}^{2} g_{y}\right]_{0} \\
& +20\left[D_{0} g\right]_{0} \cdot\left[D_{0}^{3} g_{y}\right]_{0}+15 g_{0} \cdot\left[D_{0}^{4} g_{y}\right]_{0}+\left[D_{0}^{2} g\right]_{0} \cdot g_{y, 0}^{2}+18 g_{0} \cdot\left[D_{0} g_{y}\right]_{0}^{2} \\
& +10\left[D_{0} g\right]_{0} \cdot g_{y, 0} \cdot\left[D_{0} g_{y}\right]_{0}+21 g_{0} \cdot g_{y, 0} \cdot\left[D_{0}^{2} g_{y}\right]_{0}+10\left[D_{0} g\right]_{0}^{2} \cdot g_{y y, 0} \\
& +15 g_{0} \cdot\left[D_{0}^{2} g\right]_{0} \cdot g_{y y, 0}+60 g_{0} \cdot\left[D_{0} g\right]_{0} \cdot\left[D_{0} g_{y y}\right]_{0}+45 g_{0}^{2} \cdot\left[D_{0}^{2} g_{y y}\right]_{0} \\
& +g_{0} \cdot g_{y, 0}^{3}+18 g_{0}^{2} \cdot g_{y, 0} \cdot g_{y y, 0}+15 g_{0}^{3} \cdot g_{y y y, 0} \\
& \kappa_{7}=\left[D_{0}^{7} g\right]_{0}+\left[D_{0}^{5} g\right] \cdot g_{y, 0}+7\left[D_{0}^{4} g\right]_{0} \cdot\left[D_{0} g_{y}\right]_{0}+21\left[D_{0}^{3} g\right]_{0} \cdot\left[D_{0}^{2} g_{y}\right]_{0} \\
& +\left[D_{0}^{3} g\right]_{0} \cdot g_{y, 0}^{2}+35\left[D_{0}^{2} g\right]_{0} \cdot\left[D_{0}^{3} g_{y}\right]_{0}+12\left[D_{0}^{2} g\right]_{0} \cdot g_{y, 0} \cdot\left[D_{0} g_{y}\right]_{0} \\
& +35\left[D_{0} g\right]_{0} \cdot\left[D_{0}^{4} g_{y}\right]_{0}+28\left[D_{0} g\right]_{0} \cdot\left[D_{0} g_{y}\right]_{0}^{2}+31\left[D_{0} g\right]_{0} \cdot g_{y, 0} \cdot\left[D_{0}^{2} g_{y}\right]_{0} \\
& +\left[D_{0} g\right]_{0} \cdot g_{y, 0}^{3}+35\left[D_{0} g\right]_{0} \cdot\left[D_{0}^{2} g\right]_{0} \cdot g_{y y, 0}+70\left[D_{0} g\right]_{0}^{2} \cdot\left[D_{0} g_{y y}\right]_{0} \\
& +21 g_{0} \cdot\left[D_{0}^{5} g_{y}\right]_{0}+105 g_{0} \cdot\left[D_{0} g_{y}\right]_{0} \cdot\left[D_{0}^{2} g_{y}\right]_{0}+45 g_{0} \cdot g_{y, 0} \cdot\left[D_{0}^{3} g_{y}\right]_{0} \\
& +15 g_{0} \cdot g_{y, 0}^{2} \cdot\left[D_{0} g_{y}\right]_{0}+21 g_{0} \cdot\left[D_{0}^{3} g\right]_{0} \cdot g_{y y, 0}+105 g_{0} \cdot\left[D_{0}^{2} g\right]_{0} \cdot\left[D_{0} g_{y y}\right]_{0} \\
& +210 g_{0} \cdot\left[D_{0} g\right]_{0} \cdot\left[D_{0}^{2} g_{y y}\right]_{0}+66 g_{0} \cdot\left[D_{0} g\right]_{0} \cdot g_{y, 0} \cdot g_{y y, 0}+105 g_{0}^{2} \cdot\left[D_{0}^{3} g_{y y}\right]_{0} \\
& +84 g_{0}^{2} \cdot\left[D_{0} g_{y}\right]_{0} \cdot g_{y y, 0}+120 g_{0}^{2} \cdot g_{y, 0} \cdot\left[D_{0} g_{y y}\right]_{0}+105 g_{0}^{2} \cdot\left[D_{0} g\right]_{0} \cdot g_{y y y, 0} \\
& +105 g_{0}^{3} \cdot\left[D_{0} g_{y y y}\right]_{0} \\
& \kappa_{8}=\left[D_{0}^{8} g\right]_{0}+\left[D_{0}^{6} g\right]_{0} \cdot g_{y, 0}+8\left[D_{0}^{5} g\right]_{0} \cdot\left[D_{0} g_{y}\right]_{0}+28\left[D_{0}^{4} g\right]_{0} \cdot\left[D_{0}^{2} g_{y}\right]_{0} \\
& +\left[D_{0}^{4} g\right]_{0} \cdot g_{y, 0}^{2}+56\left[D_{0}^{3} g\right]_{0} \cdot\left[D_{0}^{3} g_{y}\right]_{0}+14\left[D_{0}^{3} g\right]_{0} \cdot g_{y, 0} \cdot\left[D_{0} g_{y}\right]_{0}
\end{aligned}
$$




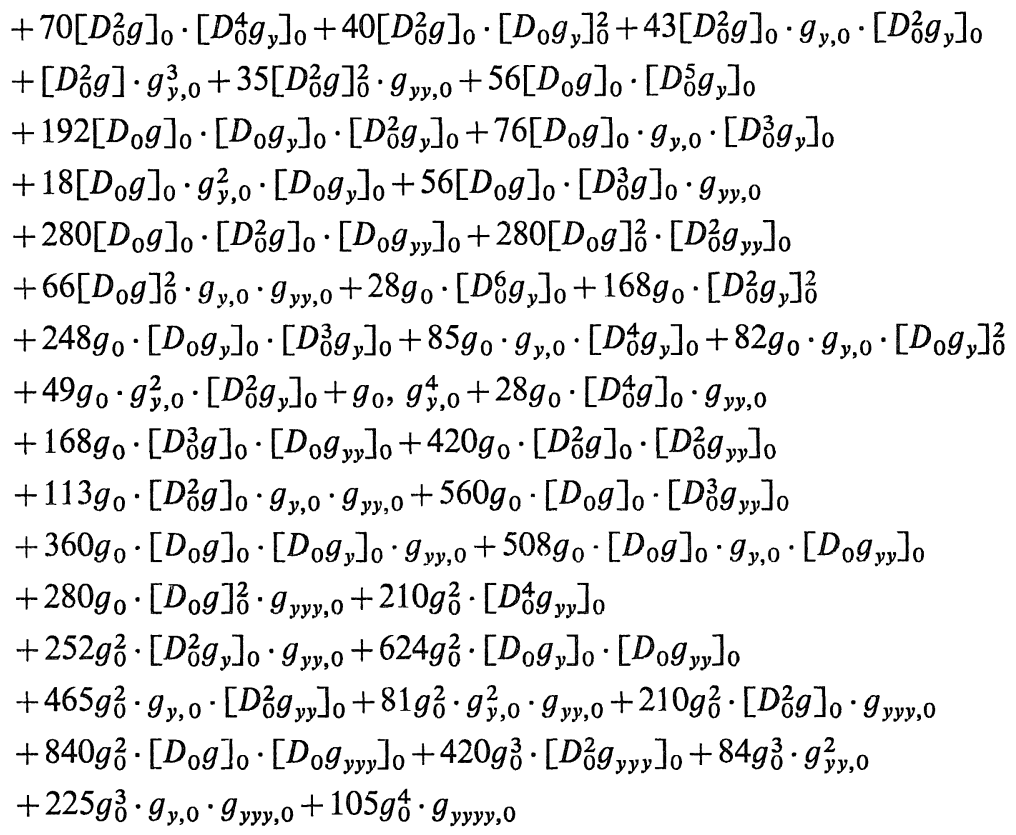

\section{§1.3. Implicit (1, q)-Stage Formula}

For the study of the general $(1, q)$-stage formula, it is convenient to analyse the corresponding implicit $(1, q)$-stage formula, because we gain an insight into its algebraic relations by them.

Consider an implicit $(1, q)$-stage formula as follows:

$$
\begin{gathered}
y_{n+1}=y_{n}+\mu_{1} h k_{1}+\sum_{i=1}^{q} v_{i} h^{2} K_{i}, \quad n=0,1,2, \ldots, \\
\left\{\begin{array}{l}
k_{1}=f\left(x_{n}+\alpha_{1} h, y_{0}\right), \\
K_{i}=g\left(x_{n}+\rho_{i} h, y_{n}+\sigma_{i 1} h k_{1}+\sum_{j=1}^{q} \tau_{i j} h^{2} K_{j}\right), \quad i=1, \ldots, q .
\end{array}\right.
\end{gathered}
$$

We need to analyse one-step integration by (1) and (2), so we may substitute $n=0$, i.e.

$$
\left\{\begin{array}{l}
y_{1}=y_{0}+\mu_{1} h k_{1}+\sum_{i=1}^{q} v_{i} h^{2} K_{i}, \\
k_{1}=f\left(x_{0}+\alpha_{1} h, y_{0}\right), \\
K_{i}=g\left(x_{0}+\rho_{i} h, y_{0}+\sigma_{i 1} h k_{1}+\sum_{j} \tau_{i j} h^{2} K_{j}\right), \quad i=1, \ldots, q .
\end{array}\right.
$$

For $k_{1}$, we have the following expansion: 


$$
k_{1}=f_{0}+\alpha_{1} h f_{x, 0}+\frac{1}{2} \alpha_{1}^{2} h^{2} f_{x x, 0}+O\left(h^{3}\right) .
$$

On the other hand, $K_{i}$ has the expansion

$$
K_{l}=g_{0}+O(h) \text {. }
$$

Hence, the equation

$$
y_{1}=y_{0}+\mu_{1} h f_{0}+h^{2}\left(\mu_{1} \alpha_{1} f_{x, 0}+\sum_{i=1}^{q} v_{i} g_{0}\right)+O\left(h^{3}\right)
$$

holds. The solution $y\left(x_{0}+h\right)$ has the power series of $h$ as follows:

$$
y\left(x_{0}+h\right)=y_{0}+h f_{0}+\frac{1}{2} h^{2} g_{0}+O\left(h^{3}\right) .
$$

Comparison of the terms having the same power of $h$ in (4) and (5) yields the following equations.

$$
\begin{gathered}
\mu_{1}=1, \\
\mu_{1} \alpha_{1} f_{x, 0}+\sum_{i=1}^{q} v_{i} g_{0}=\frac{1}{2} g_{0} .
\end{gathered}
$$

Thus, $\mu_{1}=1, \alpha_{1}=0$ and $\sum_{i=1}^{q} v_{i}=1 / 2$ must hold in the implicit $(1, q)$-stage formula.

Next, since $K_{i}$ has the expansion

$$
K_{i}=g_{0}+\left[\left(\rho_{i} \frac{\partial}{\partial x}+\sigma_{i 1} f_{0} \frac{\partial}{\partial y}\right) g\right]_{0} \cdot h+O\left(h^{2}\right),
$$

the comparison of the third order term of $y_{1}$ and $y\left(x_{0}+h\right)$ implies the equation

$$
\frac{1}{2}\left[D_{0} g\right]_{0}=\frac{1}{2}\left(g_{x, 0}+f_{0} \cdot g_{y, 0}\right)=\sum_{i=1}^{q} v_{i}\left[\left(\rho_{i} g_{x, 0}+\sigma_{i 1} f_{0} \cdot g_{y, 0}\right)\right] \text {. }
$$

From the viewpoint of homogenuity of differential operation, $\rho_{i}=\sigma_{i 1}$ must hold for $i=1,2, \ldots, q$.

Hence, let us rewrite (3) by

$$
\left\{\begin{array}{l}
y_{1}=y_{0}+h f_{0}+\sum_{i=1}^{q} v_{i} h^{2} K_{i}, \\
K_{i}=g\left(x_{0}+\rho_{i} h, y_{0}+\rho_{i} h f_{0}+\sum_{j} \tau_{i j} h^{2} K_{j}\right), \quad i=1, \ldots, q .
\end{array}\right.
$$

We shall assume the Taylor series expansion for $K_{i}$ by

$$
K_{i}=\sum_{l=0}^{m} \frac{\kappa_{i l}}{l !} h^{l}+O\left(h^{m+1}\right),
$$

where $\kappa_{i l}$ will be determined by the similar manner for Theorem 1 . 
Theorem 3. For each $i, \kappa_{i l}$ in (1.3.7) is determined by the following:

$$
\left\{\begin{array}{l}
\kappa_{i 0}=g_{0}, \\
\kappa_{i l}=\rho_{i}^{l}\left[D_{0}^{l} g\right]_{0}+\sum_{s=1}^{l-1}\left\{\sum_{t=1}^{\mathrm{m}(l, s)} \frac{l !}{s !(l-s-t) !} \rho_{i}^{l-s-t}\right. \\
\left.\left.\times B_{s, t}\left(\sum_{j=1}^{q} \tau_{i j} \kappa_{j 0}, \ldots, s \sum_{j} \tau_{i j} \kappa_{j, s-1}\right)\left[D_{0}^{l-s-t}\left(\frac{\partial}{\partial y}\right)^{t} g\right]\right]_{0}\right\} \\
\quad l=1,2, \ldots, m
\end{array}\right.
$$

Note. By the above formula, $\kappa_{i l}$ can be determined in the ascending order of the second subscript, i.e., $\kappa_{i 2}(i=1, \ldots, q)$ after $\kappa_{i 1}, \kappa_{i 3}(i=1, \ldots, q)$ after $\kappa_{i 2}$, and so on.

Proof. Two-variable Taylor series expansion for $K_{i}$ gives

$$
\begin{aligned}
K_{i} & =\sum_{l=0}^{m} \frac{1}{l !}\left[\left\{\rho_{i} h \frac{\partial}{\partial x}+\left(\rho_{i} h f_{0}+\sum \tau_{i j} h^{2} K_{j}\right) \frac{\partial}{\partial y}\right\}^{l} g\right]_{0}+O\left(h^{m+1}\right) \\
& =\sum_{l=0}^{m} \frac{h^{l}}{l !}\left[\left\{\rho_{i} D_{0}+\left(\sum \tau_{i j} h K_{j}\right) \frac{\partial}{\partial y}\right\}^{l} g\right]_{0}+O\left(h^{m+1}\right) \\
& =\sum_{l=0}^{m} \frac{h^{l}}{l !}\left[\left\{\rho_{i} D_{0}+\left(\sum_{r=0}^{m-1}\left(\sum_{j=1}^{q} \frac{\tau_{i j} \kappa_{j r}}{r !}\right) h^{r+1}\right) \frac{\partial}{\partial y}\right\}^{l} g\right]_{0}+O\left(h^{m+1}\right) \\
& =\sum_{l=0}^{m} \frac{h^{l}}{l !}\left\{\sum_{k=0}^{l}\left(\begin{array}{c}
l \\
k
\end{array}\right) \rho_{i}^{l-k}\left(\sum_{r=0}^{m-1}\left(\sum_{j} \frac{\tau_{i j} \kappa_{j r}}{r !}\right) h^{r+1}\right)^{k}\left[D_{0}^{l-k}\left(\frac{\partial}{\partial y}\right)^{k} g\right]_{0}\right\} \\
& +O\left(h^{m+1}\right) .
\end{aligned}
$$

Thus, putting $G_{i}(h)$ as

$$
G_{i}(h)=\sum_{r=0}^{m-1}\left(\sum_{j=1}^{q} \frac{\tau_{i j} \kappa_{j r}}{r !}\right) h^{r+1}
$$

and noting that $G_{i}(h)$ is of the order $h$ and $G_{i}(0)=0$, we have the equation

$$
\sum_{l=0}^{m} \frac{\kappa_{i l}}{l !} h^{l}=\sum_{l=0}^{m} \frac{h^{l}}{l !}\left\{\sum_{k=0}^{l}\left(\begin{array}{c}
l \\
k
\end{array}\right) \rho_{i}^{l-k}\left(G_{i}(h)\right)^{k}\left[D_{0}^{l-k}\left(\frac{\partial}{\partial y}\right)^{k} g\right]_{0}\right\},
$$

which is similar to (1.2.4). Hence, following after the proof of Theorem 1, we have

$$
\begin{array}{r}
\kappa_{i l}=\rho_{i}^{l}\left[D_{0}^{l} g\right]_{0}+\sum_{s=1}^{l-1}\left(\begin{array}{l}
l \\
s
\end{array}\right)\left\{\sum_{i=1}^{\mathrm{m}(l, s)} \frac{(l-s) !}{(l-s-t) !} \rho_{i}^{l-s-t} B_{s, t}\left(G_{i}^{\prime}(0), \ldots, G_{i}^{(s)}(0)\right)\right. \\
\left.\times\left[D_{0}^{l-s-t}\left(\frac{\partial}{\partial y}\right)^{t} g\right]_{0}\right\} .
\end{array}
$$

From (9), for $k=1,2, \ldots, s$

$$
G_{i}^{(k)}(h)=\sum_{r=k-1}^{m-1} \frac{(r+1) !}{(r-k+1) !}\left(\sum_{j} \frac{\tau_{i j} \kappa_{j_{r}}}{r !}\right) h^{r-k+1}
$$


holds, which implies

$$
G_{i}^{(k)}(0)=k !\left(\sum_{j} \frac{\tau_{i j} \kappa_{j, k-1}}{(k-1) !}\right)=k \sum_{j} \tau_{i j} \kappa_{j, k-1} .
$$

Thus we have the desired result.

By Theorem 3, $\kappa_{i l}$ is given as follows:

$$
\begin{aligned}
\kappa_{i 1} & =\rho_{i}\left[D_{0} g\right]_{0}, \\
\kappa_{i 2} & =\rho_{i}^{2}\left[D_{0}^{2} g\right]_{0}+2 ! B_{1,1}\left(\sum_{j} \tau_{i j} \kappa_{j 0}\right) \cdot g_{y, 0} \\
& =\rho_{i}^{2}\left[D_{0}^{2} g\right]_{0}+2\left(\sum_{j} \tau_{i j}\right) g_{0} \cdot g_{y, 0}, \\
\kappa_{i 3} & =\rho_{i}^{3}\left[D_{0}^{3} g\right]_{0}+3 ! \rho_{i} \cdot B_{1,1}\left(\sum \tau_{i j} \kappa_{j 0}\right)\left[D_{0} g_{y}\right]_{0}+3 B_{2,1}\left(\sum \tau_{i j} \kappa_{j 0}, 2 \sum \tau_{i j} \kappa_{j 1}\right) \cdot g_{y, 0} \\
& =\rho_{i}^{3}\left[D_{0}^{3} g\right]_{0}+6 \rho_{i}\left(\sum_{j} \tau_{i j}\right) g_{0} \cdot\left[D_{0} g_{y}\right]_{0}+6\left(\sum_{j} \tau_{i j} \rho_{j}\right)\left[D_{0} g\right]_{0} \cdot g_{y, 0} \cdot
\end{aligned}
$$

The result with the help of REDUCE-2 is shown in Table 2. Here we employ the notation

$$
T_{i k}=\sum_{j=1}^{q} \rho_{j}^{k} \tau_{i j}, \quad i=1,2, \ldots, q, \quad k=0,1,2, \ldots
$$

From (6) and (7), the Taylor series expansion for $y_{1}$ is given by

$$
\begin{aligned}
y_{1} & =y_{0}+h f_{0}+\sum_{i=1}^{q} v_{i} h^{2}\left(\sum_{l=0}^{m} \frac{\kappa_{i l}}{l !} h^{l}\right)+O\left(h^{m+3}\right) \\
& =y_{0}+h f_{0}+\sum_{l=0}^{m}\left(\sum_{i=1}^{q} v_{i} \kappa_{i l}\right) \frac{h^{l+2}}{l !}+O\left(h^{m+3}\right) \\
& =y_{0}+h f_{0}+\sum_{r=2}^{m}\left(\sum_{i=1}^{q} v_{i} \kappa_{i, r-2}\right) \frac{h^{r}}{(r-2) !}+O\left(h^{m+1}\right) .
\end{aligned}
$$

On the other hand, from (1.2.2), the (second type) Taylor series expansion of the solution $y\left(x_{0}+h\right)$ is given by

$$
y\left(x_{0}+h\right)=y_{0}+h f_{0}+\sum_{r=2}^{m} \frac{\kappa_{r-2}}{r !} h^{r}+O\left(h^{m+1}\right),
$$

where $\kappa_{l}$ is represented by (1.2.5). The comparison of (10) and (11) brings the determining equation of the implicit $(1, q)$-stage formula, for which we have the following

Theorem 4. Each $\kappa_{i l}$ in (10) is constructed with the all elementary differentials included in the corresponding $\kappa_{l}$ in (11). Hence the determining equation of the implicit $(1, q)$-stage formula is of the form such as

(an integer $) \times\left(\right.$ a polynomial of $\left.v_{i}, \rho_{i}, \tau_{i j}\right)=($ an integer $)$. 
Proof. From (10) and (11), $\kappa_{l}$ and $\kappa_{i, l}$ must satisfy

$$
\frac{1}{l !} \sum_{i=1}^{q} v_{i} \kappa_{i l}=\frac{\kappa_{l}}{(l+2) !}, \quad l=0,1,2, \ldots, m-2,
$$

which implies

$$
(l+1)(l+2) \sum_{i} v_{i} \kappa_{i l}=\kappa_{l} .
$$

From (1.2.5) and (1.3.8) the conclusion follows.

\section{Table 2.}

$$
\begin{aligned}
& \kappa_{i 0}=g_{0} \\
& \kappa_{i 1}=\rho_{i}\left[D_{0} g\right]_{0} \\
& \kappa_{i 2}=\rho_{i}^{2}\left[D_{0}^{2} g\right]_{0}+2 T_{i 0} \cdot g_{0} \cdot g_{y, 0} \\
& \kappa_{i 3}=\rho_{i}^{3}\left[D_{0}^{3} g\right]_{0}+6 T_{i 1} \cdot\left[D_{0} g\right]_{0} \cdot g_{y, 0}+6 \rho_{i} T_{i 0} \cdot g_{0} \cdot\left[D_{0} g_{y}\right]_{0} \\
& \kappa_{i 4}=\rho_{i}^{4}\left[D_{0}^{4} g\right]_{0}+12 T_{i 2}\left[D_{0}^{2} g\right]_{0} \cdot g_{y, 0}+24 \rho_{i} T_{i 1}\left[D_{0} g\right]_{0} \cdot\left[D_{0} g_{y}\right]_{0} \\
& +12 \rho_{i}^{2} T_{i 0} \cdot g_{0} \cdot\left[D_{0}^{2} g_{y}\right]_{0}+24\left(\sum_{j} \tau_{i j} T_{j 0}\right) g_{0} \cdot g_{y, 0}^{2}+12 T_{i 0}^{2} \cdot g_{0}^{2} \cdot g_{y y, 0} \\
& \kappa_{i 5}=\rho_{i}^{5} \cdot\left[D_{0}^{5} g\right]_{0}+20 T_{i 3} \cdot\left[D_{0}^{3} g\right]_{0} \cdot g_{y, 0}+60 \rho_{i} T_{i 2} \cdot\left[D_{0}^{2} g\right]_{0}\left[D_{0} g_{y}\right]_{0} \\
& +60 \rho_{i}^{2} T_{i 1}\left[D_{0} g\right]_{0}\left[D_{0}^{2} g_{y}\right]_{0}+120\left(\sum_{j} \tau_{i j} T_{j 1}\right) \cdot\left[D_{0} g\right]_{0} \cdot g_{y, 0}^{2} \\
& +20 \rho_{i}^{3} T_{i 0} \cdot g_{0} \cdot\left[D_{0}^{3} g_{y}\right]_{0}+120\left\{\sum_{j}\left(\rho_{i}+\rho_{j}\right) \tau_{i j} T_{j 0}\right\} g_{0} \cdot g_{y, 0} \cdot\left[D_{0} g_{y}\right]_{0} \\
& +120 T_{i 0} T_{i 1} \cdot g_{0} \cdot\left[D_{0} g\right]_{0} \cdot g_{y y, 0}+60 \rho_{i} T_{i 0}^{2} \cdot g_{0}^{2} \cdot\left[D_{0} g_{y y}\right]_{0} \\
& \kappa_{i 6}=\rho_{i}^{6}\left[D_{0}^{6} g\right]_{0}+30 T_{i 4}\left[D_{0}^{4} g\right]_{0} \cdot g_{y, 0}+120 \rho_{i} T_{i 3}\left[D_{0}^{3} g\right] \cdot\left[D_{0} g_{y}\right]_{0} \\
& +180 \rho_{i}^{2} T_{i 2}\left[D_{0}^{2} g\right]_{0}\left[D_{0}^{2} g_{y}\right]_{0}+360\left(\sum_{j} \tau_{i j} T_{j 2}\right)\left[D_{0}^{2} g\right]_{0} \cdot g_{y, 0}^{2} \\
& +120 \rho_{i}^{3} T_{i 1}\left[D_{0} g\right]_{0} \cdot\left[D_{0}^{3} g_{y}\right]_{0}+720\left\{\sum_{j}\left(\rho_{i}+\rho_{j}\right) \tau_{i j} T_{j 1}\right\}\left[D_{0} g\right]_{0} \cdot g_{y, 0} \cdot\left[D_{0} g_{y}\right]_{0} \\
& +360 T_{i 1}^{2}\left[D_{0} g\right]_{0}^{2} \cdot g_{y y, 0}+30 \rho_{i}^{4} T_{i 0} \cdot g_{0} \cdot\left[D_{0}^{4} g_{y}\right]_{0} \\
& +720\left(\sum_{j} \rho_{i} \rho_{j} \tau_{i j} T_{j 0}\right) \cdot g_{0} \cdot\left[D_{0} g_{y}\right]_{0}^{2}+360\left\{\sum_{j}\left(\rho_{i}^{2}+\rho_{j}^{2}\right) \tau_{i j} T_{j 0}\right\} g_{0} \cdot g_{y, 0} \cdot\left[D_{0}^{2} g_{y}\right]_{0} \\
& +720\left(\sum_{j} \sum_{k} \tau_{i j} \tau_{j k} T_{k 0}\right) g_{0} \cdot g_{y, 0}^{3}+360 T_{i 0} T_{i 2} \cdot g_{0} \cdot\left[D_{0}^{2} g\right]_{0} \cdot g_{y y, 0} \\
& +720 \rho_{i} T_{i 0} T_{i 1} \cdot g_{0} \cdot\left[D_{0} g\right]_{0} \cdot\left[D_{0} g_{y y}\right]_{0}+180 \rho_{i}^{2} T_{i 0}^{2} g_{0}^{2} \cdot\left[D_{0}^{2} g_{y y}\right]_{0} \\
& +360\left\{\sum_{j} \tau_{i j}\left(T_{j 0}^{2}+2 T_{i 0} T_{j 0}\right)\right\} g_{0}^{2} \cdot g_{y, 0} \cdot g_{y y, 0}+120 T_{i 0}^{3} \cdot g_{0}^{3} \cdot g_{y y y, 0} \\
& \kappa_{i 7}=\rho_{i}^{7} \cdot\left[D_{0}^{7} g\right]_{0}+42 T_{i 5}\left[D_{0}^{5} g\right]_{0} \cdot g_{y, 0}+210 \rho_{i} T_{i 4}\left[D_{0}^{4} g\right]_{0} \cdot\left[D_{0} g_{y}\right]_{0} \\
& +420 \rho_{i}^{2} T_{i 3}\left[D_{0}^{3} g\right]_{0} \cdot\left[D_{0}^{2} g_{y}\right]_{0}+840\left(\sum_{j} \tau_{i j} T_{j 3}\right)\left[D_{0}^{3} g\right]_{0} \cdot g_{y, 0}^{2} \\
& +420 \rho_{i}^{3} T_{i 2}\left[D_{0}^{2} g\right]_{0} \cdot\left[D_{0}^{3} g_{y}\right]_{0}+2520\left(\sum_{j}\left(\rho_{i}+\rho_{j}\right) \tau_{i j} T_{j 2}\right)\left[D_{0}^{2} g\right]_{0} \cdot g_{y, 0} \cdot\left[D_{0} g_{y}\right]_{0} \\
& +210 \rho_{i}^{4} T_{i 1}\left[D_{0} g\right]_{0} \cdot\left[D_{0}^{4} g_{y}\right]_{0}+5040\left(\sum_{j} \rho_{i} \rho_{j} \tau_{i j} T_{j 1}\right)\left[D_{0} g\right]_{0} \cdot\left[D_{0} g_{y}\right]_{0}^{2} \\
& +2520\left(\sum_{j}\left(\rho_{i}^{2}+\rho_{j}^{2}\right) \tau_{i j} T_{j 1}\right)\left[D_{0} g\right]_{0} \cdot g_{y, 0} \cdot\left[D_{0}^{2} g_{y}\right]_{0}
\end{aligned}
$$




$$
\begin{aligned}
& +5040\left(\sum_{j} \sum_{k} \tau_{i j} \tau_{j k} T_{k 1}\right)\left[D_{0} g\right]_{0} \cdot g_{y, 0}^{3}+2520 T_{i 1} T_{i 2}\left[D_{0} g\right]_{0} \cdot\left[D_{0}^{2} g\right]_{0} \cdot g_{y y, 0} \\
& +2520 \rho_{i} T_{i 1}^{2}\left[D_{0} g\right]_{0}^{2} \cdot\left[D_{0} g_{y y}\right]_{0}+42 \rho_{i}^{5} T_{i 0} g_{0} \cdot\left[D_{0}^{5} g_{y}\right]_{0} \\
& +2520\left(\sum_{j} \rho_{i} \rho_{j}\left(\rho_{i}+\rho_{j}\right) \tau_{i j} T_{j 0}\right) g_{0} \cdot\left[D_{0} g_{y}\right]_{0} \cdot\left[D_{0}^{2} g_{y}\right]_{0} \\
& \left.\left.+840\left(\sum_{j}\right) \rho_{i}^{3}+\rho_{j}^{3}\right) \tau_{i j} T_{j 0}\right) g_{0} \cdot g_{y, 0} \cdot\left[D_{0}^{3} g_{y}\right]_{0} \\
& +5040\left(\sum_{j} \sum_{k}\left(\rho_{i}+\rho_{j}+\rho_{k}\right) \tau_{i j} \tau_{j k} T_{k 0}\right) g_{0} \cdot g_{y, 0}^{2} \cdot\left[D_{0} g_{y}\right]_{0} \\
& +840 T_{i 0} T_{i 3} g_{0} \cdot\left[D_{0}^{3} g\right]_{0} \cdot g_{y y, 0}+2520 \rho_{i} T_{i 0} T_{i 2} g_{0} \cdot\left[D_{0}^{2} g\right]_{0} \cdot\left[D_{0} g_{y y}\right]_{0} \\
& +2520 \rho_{i}^{2} T_{i 0} T_{i 1} g_{0} \cdot\left[D_{0} g\right]_{0} \cdot\left[D_{0}^{2} g_{y y}\right]_{0} \\
& +5040\left(\sum_{j} \tau_{i j}\left(T_{i 0} T_{j 1}+T_{j 0} T_{j 1}+T_{j 0} T_{i 1}\right)\right) g_{0} \cdot\left[D_{0} g\right]_{0} \cdot g_{y, 0} \cdot g_{y y, 0} \\
& +420 \rho_{i}^{3} T_{i 0}^{2} g_{0}^{2} \cdot\left[D_{0}^{3} g_{y y}\right]_{0} \\
& +2520\left(\sum_{j}\left(2 \rho_{i} T_{i 0}+\rho_{j} T_{j 0}\right) \tau_{i j} T_{j 0}\right) g_{0}^{2} \cdot\left[D_{0} g_{y}\right]_{0} \cdot g_{y y, 0} \\
& +2520\left(\sum_{j}\left(\rho_{i} T_{j 0}+2 \rho_{j} T_{i 0}\right) \tau_{i j} T_{j 0}\right) g_{0}^{2} \cdot g_{y, 0} \cdot\left[D_{0} g_{y y}\right]_{0} \\
& +2520 T_{i 0}^{2} T_{i 1} g_{0}^{2} \cdot\left[D_{0} g\right]_{0} \cdot g_{y y y, 0}+840 \rho_{i} T_{i 0}^{3} g_{0}^{3} \cdot\left[D_{0} g_{y y y}\right]_{0} \\
& \kappa_{i 8}=\rho_{i}^{8}\left[D_{0}^{8} g\right]_{0}+56 T_{i 6} \cdot\left[D_{0}^{6} g\right]_{0} \cdot g_{y, 0}+336 \rho_{i} T_{i 5}\left[D_{0}^{5} g\right]_{0} \cdot\left[D_{0} g_{y}\right]_{0} \\
& +840 \rho_{i}^{2} T_{i 4}\left[D_{0}^{4} g\right]_{0} \cdot\left[D_{0}^{2} g_{y}\right]_{0}+1680\left(\sum_{j} \tau_{i j} T_{j 4}\right)\left[D_{0}^{4} g\right]_{0} \cdot g_{y, 0}^{2} \\
& +1120 \rho_{i}^{3} T_{\imath 3}\left[D_{0}^{3} g\right]_{0} \cdot\left[D_{0}^{3} g_{y}\right]_{0}+6720\left(\sum_{j}\left(\rho_{i}+\rho_{j}\right) \tau_{i j} T_{j 3}\right)\left[D_{0}^{3} g\right]_{0} \cdot g_{y, 0} \cdot\left[D_{0} g_{y}\right]_{0} \\
& +840 \rho_{i}^{4} T_{i 2}\left[D_{0}^{2} g\right]_{0} \cdot\left[D_{0}^{4} g_{y}\right]_{0}+20160\left(\sum_{j} \rho_{i} \rho_{j} \tau_{i j} T_{j 2}\right)\left[D_{0}^{2} g\right]_{0} \cdot\left[D_{0} g_{y}\right]_{0}^{2} \\
& +10080\left(\sum_{j}\left(\rho_{i}^{2}+\rho_{j}^{2}\right) \tau_{i j} T_{j 2}\right)\left[D_{0}^{2} g\right]_{0} \cdot g_{y, 0} \cdot\left[D_{0}^{2} g_{y}\right]_{0} \\
& +20160\left(\sum_{j}^{j} \sum_{k} \tau_{i j} \tau_{j k} T_{k 2}\right)\left[D_{0}^{2} g\right]_{0} \cdot g_{y, 0}^{3}+5040 T_{i 2}^{2}\left[D_{0}^{2} g\right]_{0}^{2} \cdot g_{y y, 0} \\
& +336 \rho_{i}^{5} T_{i 1}\left[D_{0} g\right]_{0} \cdot\left[D_{0}^{5} g_{y}\right]_{0} \\
& +20160\left(\sum_{j} \rho_{i} \rho_{j}\left(\rho_{i}+\rho_{j}\right) \tau_{i j} T_{j 1}\right)\left[D_{0} g\right]_{0} \cdot\left[D_{0} g_{y}\right]_{0} \cdot\left[D_{0}^{2} g_{y}\right]_{0} \\
& +6720\left(\sum_{j}\left(\rho_{i}^{3}+\rho_{j}^{3}\right) \tau_{i j} T_{j 1}\right)\left[D_{0} g\right]_{0} \cdot g_{y, 0} \cdot\left[D_{0}^{3} g_{y}\right]_{0} \\
& +40320\left(\sum_{j} \sum_{k}\left(\rho_{i}+\rho_{j}+\rho_{k}\right) \tau_{i j} \tau_{j k} T_{k 1}\right)\left[D_{0} g\right]_{0} \cdot g_{y, 0}^{2} \cdot\left[D_{0} g_{y}\right]_{0} \\
& +6720 T_{i 1} T_{i 3}\left[D_{0} g\right]_{0} \cdot\left[D_{0}^{3} g\right]_{0} \cdot g_{y y, 0}+20160 \rho_{i} T_{i 1} T_{i 2}\left[D_{0} g\right]_{0} \cdot\left[D_{0}^{2} g\right]_{0} \cdot\left[D_{0} g_{y y}\right]_{0} \\
& +10080 \rho_{i}^{2} T_{i 1}^{2}\left[D_{0} g\right]_{0}^{2} \cdot\left[D_{0}^{2} g_{y y}\right]_{0} \\
& +20160\left(\sum_{j} \tau_{i j} T_{j 1}\left(2 T_{i 1}+T_{j 1}\right)\right)\left[D_{0} g\right]_{0}^{2} \cdot g_{y, 0} \cdot g_{y y, 0} \\
& +56 \rho_{i}^{6} T_{i 0} g_{0} \cdot\left[D_{0}^{6} g_{y}\right]_{0}+10080\left(\sum_{j} \rho_{i}^{2} \rho_{j}^{2} \tau_{i j} T_{j 0}\right) g_{0} \cdot\left[D_{0}^{2} g_{y}\right]_{0}^{2} \\
& +6720\left(\sum_{j} \rho_{i} \rho_{j}\left(\rho_{i}^{2}+\rho_{j}^{2}\right) \tau_{i j} T_{j 0}\right) g_{0} \cdot\left[D_{0} g_{y}\right]_{0} \cdot\left[D_{0}^{3} g_{y}\right]_{0} \\
& +1680\left(\sum_{j}\left(\rho_{i}^{4}+\rho_{j}^{4}\right) \tau_{i j} T_{j 0}\right) g_{0} \cdot g_{y, 0} \cdot\left[D_{0}^{4} g_{y}\right]_{0} \\
& +40320\left(\sum_{j} \sum_{k}\left(\rho_{i} \rho_{j}+\rho_{j} \rho_{k}+\rho_{i} \rho_{k}\right) \tau_{i j} \tau_{j k} T_{k 0}\right) g_{0} \cdot g_{y, 0} \cdot\left[D_{0} g_{y}\right]_{0}^{2} \\
& +20160\left(\sum_{j} \sum_{k}\left(\rho_{i}^{2}+\rho_{j}^{2}+\rho_{k}^{2}\right) \tau_{i j} \tau_{j k} T_{k 0}\right) g_{0} \cdot g_{y, 0}^{2} \cdot\left[D_{0}^{2} g_{y}\right]_{0} \\
& +40320\left(\sum_{j} \sum_{k} \sum_{l} \tau_{i j} \tau_{j k} \tau_{k l} T_{l 0}\right) g_{0} \cdot g_{y, 0}^{4}+1680 T_{i 0} T_{i 4} g_{0} \cdot\left[D_{0}^{4} g\right]_{0} \cdot g_{y y, 0}
\end{aligned}
$$




$$
\begin{aligned}
& +6720 \rho_{i} T_{i 0} T_{i 3} g_{0} \cdot\left[D_{0}^{3} g\right]_{0} \cdot\left[D_{0} g_{y y}\right]_{0} \\
& +10080 \rho_{i}^{2} T_{i 0} T_{i 2} g_{0} \cdot\left[D_{0}^{2} g\right]_{0} \cdot\left[D_{0}^{2} g_{y y}\right]_{0} \\
& +20160\left(\sum_{j} \tau_{i j}\left(T_{i 2} T_{j 0}+T_{i 0} T_{j 2}+T_{j 0} T_{j 2}\right)\right) g_{0} \cdot\left[D_{0}^{2} g\right]_{0} \cdot g_{y, 0} \cdot g_{y y, 0} \\
& +6720 \rho_{i}^{3} T_{i 0} T_{i 1} g_{0} \cdot\left[D_{0} g\right]_{0} \cdot\left[D_{0}^{3} g_{y y}\right]_{0} \\
& +40320\left(\sum_{j} \tau_{i j}\left(\rho_{j} T_{i 0} T_{j 1}+\rho_{j} T_{i 1} T_{j 0}+\rho_{i} T_{j 0} T_{j 1}\right)\right) g_{0} \cdot\left[D_{0} g\right]_{0} \cdot\left[D_{0} g_{y}\right]_{0} \cdot g_{y y, 0} \\
& +40320\left(\sum_{j} \tau_{i j}\left(\rho_{i} T_{i 0} T_{j 1}+\rho_{i} T_{i 1} T_{j 0}+\rho_{j} T_{j 0} T_{j 1}\right)\right) g_{0} \cdot\left[D_{0} g\right]_{0} \cdot g_{y, 0} \cdot\left[D_{0} g_{y y}\right]_{0} \\
& +20160 T_{i 0} T_{i 1}^{2} g_{0} \cdot\left[D_{0} g\right]_{0}^{2} \cdot g_{y y y, 0}+840 \rho_{i}^{4} T_{i 0}^{2} g_{0}^{2} \cdot\left[D_{0}^{4} g_{y y}\right]_{0} \\
& +10080\left(\sum_{j} \tau_{i j}\left(\rho_{i}^{2} T_{j 0}+2 \rho_{j}^{2} T_{i 0}\right) T_{j 0}\right) g_{0}^{2} \cdot\left[D_{0}^{2} g_{y}\right]_{0} \cdot g_{y y, 0} \\
& +20160\left(\sum_{j} \rho_{i} \rho_{j} \tau_{i j}\left(2 T_{i 0}+T_{j 0}\right) T_{j 0}\right) g_{0}^{2} \cdot\left[D_{0} g_{y}\right]_{0} \cdot\left[D_{0} g_{y y}\right]_{0} \\
& +10080\left(\sum_{j} \tau_{i j}\left(\rho_{j}^{2} T_{j 0}+2 \rho_{i}^{2} T_{i 0}\right) T_{j 0}\right) g_{0}^{2} \cdot g_{y, 0} \cdot\left[D_{0}^{2} g_{y y}\right]_{0} \\
& +100160\left(\sum_{j} \sum_{k} \tau_{i j}\left(2 \tau_{j k} T_{i 0} T_{k 0}+2 \tau_{j k} T_{j 0} T_{k 0}+\tau_{i k} T_{j 0} T_{k 0}+\tau_{j k} T_{k 0}^{2}\right)\right) g_{0}^{2} \cdot g_{y, 0}^{2} \cdot g_{y j, 0} \\
& +3360 \rho_{i}^{2} T_{i 0}^{3} g_{0}^{3} \cdot\left[D_{0}^{2} g_{y y y}\right]_{0}+20160\left(\sum_{j}^{2} \tau_{i j} T_{i 0} T_{j 0}^{2}\right) g_{0}^{3} \cdot g_{y y, 0}^{2} \\
& +6720\left(\sum_{j} \tau_{i j}\left(3 T_{i 0}^{2}+T_{j 0}^{2}\right) T_{j 0}\right) g_{0}^{3} \cdot g_{y, 0} \cdot g_{y y y, 0} \\
& +1680 T_{i 0}^{4} g_{0}^{4} \cdot g_{y y y y, 0}
\end{aligned}
$$

\section{§1.4. Determining Equation for $(1, q)$-Stage Formula}

An algebraic computation according to Theorem 1 gives the number of elementary differentials including in each $\kappa_{l}$. Let $m_{l}$ be the number of elementary differentials in $\kappa_{l}$ and define the integer $M_{l}$ by

$$
M_{l}=\sum_{j=0}^{l} m_{j}
$$

Each $m_{l}$ and $M_{l}$ are given in Table 3 up to $l=8$.

Thus, we have $M_{l}$ restrictions for the parameters $v_{i}, \rho_{i}, \tau_{i j}$ of the formula. In the implicit $(1, q)$-stage formula the number of the parameters to be determined, say $N_{q}^{(I)}$, can be given as a simple function of $q$

$$
N_{q}^{(I)}=q(q+2) \text {. }
$$

It implies that the implicit $(1, q)$-stage formula can attain at least the order $(\bar{l}+2)$ where $\bar{l}$ is the largest integer satisfying the inequality $M_{l} \leqq N_{q}^{(I)}$. These relations are shown in Table 4.

However, the above argument based on merely counting the number of the equations that must be satisfied, ignores the relationship between them. 
In fact, it may happen that $M_{l}$ restrictions are satisfied with fewer than $M_{l}$ variables. But, since we are interested in the explicit formula rather than implicit one, we shall not come into more investigation for the attainable order of the implicit formula.

An explicit $(1, q)$-stage formula, which is defined by the parameters $v_{i}, \rho_{i}$ and $\tau_{i j}\left(\tau_{i j}=0\right.$ for $\left.j \geqq i\right)$ in (1.3.6), has $N_{q}^{(E)}$ parameters to be determined. Here $N_{q}^{(E)}$ is given by

$$
N_{q}^{(E)}=q(q+3) / 2
$$

Hence, similar consideration for the implicit case gives the largest integer $l^{*}$ satisfying the inequality $M_{l} \leqq N_{q}^{(E)}$. Table 4 includes the relations between $q$, the number of stages, and $l^{*}$.

Table 3.

\begin{tabular}{rrr}
\hline \hline$l$ & $m_{l}$ & $M_{l}$ \\
\hline 0 & 1 & 1 \\
1 & 1 & 2 \\
2 & 2 & 4 \\
3 & 3 & 7 \\
4 & 6 & 13 \\
5 & 9 & 22 \\
6 & 17 & 39 \\
7 & 26 & 65 \\
8 & 46 & 111 \\
\hline
\end{tabular}

Table 4.

\begin{tabular}{r||rl||rr}
\hline \hline$q$ & $N_{q}^{(I)}$ & $T$ & $N_{q}^{(E)}$ & $l^{*}$ \\
\hline 1 & 3 & 1 & 2 & 1 \\
2 & 8 & 3 & 5 & 2 \\
3 & 15 & 4 & 9 & 3 \\
4 & 24 & 5 & 14 & 4 \\
5 & 35 & 5 & 20 & 4 \\
6 & 48 & 6 & 27 & 5 \\
7 & 63 & 6 & 35 & 5 \\
8 & 80 & 7 & 44 & 6 \\
9 & 99 & 7 & 54 & 6 \\
10 & 120 & 8 & 65 & 7 \\
\hline
\end{tabular}


SHINTANI has given some explicit one-step methods utilizing the second derivative [12], whose formulation coincides with our explicit $(1, q)$-stage formula. He has determined the parameters for $q=1,2,3,4$ and 5 which give the formula obtaining the order $3,4,5,6$ and 7 respectively. His results attain the orders that we have argued as the least number $l^{*}$ for each stage formula. Hence, it is a question whether Shintani's results can be improved.

We shall consider the determining equation for the explicit $(1, q)$-stage formula. Tables 1 and 2 give the equation as follows. We employ the notation for summation symbol such that the upper limit of summation can not exceed the variable of the preceding summation symbols, i.e. $\sum_{i} \cdots\left(\sum_{j} \cdots\right)$ means $\sum_{i=1}^{q} \cdots$ $\left(\sum_{j=1}^{i-1} \cdots\right)$. Moreover, the symbol $T_{i k}$ means

$$
T_{1 k}=0 \quad \text { and } \quad T_{i k}=\sum_{j=1}^{i-1} \rho_{i}^{k} \tau_{i j}, \quad i=2,3, \ldots, q .
$$

Determining equations.

$$
\begin{array}{lll}
l=0: & (\mathrm{E}-0) \quad 2 \sum_{i} v_{i}=1 \\
l=1: & (\mathrm{E}-1) \quad 6 \sum_{i} v_{i} \rho_{i}=1 \\
l=2: & (\mathrm{E}-21) \quad 12 \sum_{i} v_{i} \rho_{i}^{2}=1 \\
& (\mathrm{E}-22) \quad 24 \sum_{i} v_{i} T_{i 0}=1 \\
l=3: & (\mathrm{E}-31) & 20 \sum_{i} v_{i} \rho_{i}^{3}=1 \\
& (\mathrm{E}-32) & 120 \sum_{i} v_{i} T_{i 1}=1 \\
& (\mathrm{E}-33) \quad 120 \sum_{i} v_{i} \rho_{i} T_{i 0}=3 \\
l=4: & (\mathrm{E}-41) \quad 30 \sum_{i} v_{i} \rho_{i}^{4}=1 \\
& (\mathrm{E}-42) \quad 360 \sum_{i} v_{i} T_{i 2}=1 \\
& (\mathrm{E}-43) \quad 720 \sum_{i} v_{i} \rho_{i} T_{i 1}=4 \\
& (\mathrm{E}-44) \quad 360 \sum_{i} v_{i} \rho_{i}^{2} T_{i 0}=6 \\
& (\mathrm{E}-45) \quad 720 \sum_{i} \sum_{j} v_{i} \tau_{i j} T_{j 0}=1 \\
& (\mathrm{E}-46) \quad 360 \sum_{i} v_{i} T_{i 0}^{2}=3 \\
l=5: & (\mathrm{E}-51) \quad 42 \sum_{i} v_{i} \rho_{i}^{5}=1 \\
& (\mathrm{E}-52) \quad 840 \sum_{i} v_{i} T_{i 3}=1 \\
& (\mathrm{E}-53) \quad 2520 \sum_{i} v_{i} \rho_{i} T_{i 2}=5 \\
& (\mathrm{E}-54) \quad 2520 \sum_{i} v_{i} \rho_{i}^{2} T_{i 1}=10 \\
& (\mathrm{E}-55) \quad 5040 \sum_{i} \sum_{j} v_{i} \tau_{i j} T_{j 1}=1 \\
& (\mathrm{E}-56) \quad 840 \sum_{i} v_{i} \rho_{i}^{3} T_{i 0}=10 \\
&
\end{array}
$$




$$
\begin{aligned}
& \text { (E-57) } 5040 \sum_{i} \sum_{j} v_{i}\left(\rho_{i}+\rho_{j}\right) \tau_{i j} T_{j 0}=8 \\
& \text { (E-58) } 5040 \sum_{i} v_{i} T_{i 0} T_{i 1}=10 \\
& \text { (E-59) } 2520 \sum_{i} v_{i} \rho_{i} T_{i 0}^{2}=15 \\
& l=6: \quad(\mathrm{E}-601) \quad 56 \sum_{i} v_{i} \rho_{i}^{6}=1 \\
& \text { (E-602) } 1680 \sum_{i} v_{i} T_{i 4}=1 \\
& \text { (E-603) } 6720 \sum_{i}^{i} v_{i} \rho_{i} T_{i 3}=6 \\
& \text { (E-604) } 10080 \sum_{i} v_{i} \rho_{i}^{2} T_{i 2}=15 \\
& \text { (E-605) } 20160 \sum_{i}^{i} \sum_{j} v_{i} \tau_{i j} T_{j 2}=1 \\
& \text { (E-606) } 6720 \sum_{i} v_{i} \rho_{i}^{3} T_{i 1}=20 \\
& \text { (E-607) } 40320 \sum_{i} \sum_{j} v_{i}\left(\rho_{i}+\rho_{j}\right) \tau_{i j} T_{j 1}=10 \\
& \text { (E-608) } 20160 \sum_{i} v_{i} T_{i 1}^{2}=10 \\
& \text { (E-609) } 1680 \sum_{i} v_{i} \rho_{i}^{4} T_{i 0}=15 \\
& \text { (E-610) } 40320 \sum_{i} \sum_{j} v_{i} \rho_{i} \rho_{j} \tau_{i j} T_{j 0}=18 \\
& \text { (E-611) } 20160 \sum_{i}^{i} \sum_{j}^{j} v_{i}\left(\rho_{i}^{2}+\rho_{j}^{2}\right) \tau_{i j} T_{j 0}=21 \\
& \text { (E-612) } 40320 \sum_{i}^{i} \sum_{j} \sum_{k} v_{i} \tau_{i j} \tau_{j k} T_{k 0}=1 \\
& \text { (E-613) } 20160 \sum_{i} v_{i} T_{i 0} T_{i 2}=15 \\
& \text { (E-614) } 40320 \sum_{i}^{i} v_{i} \rho_{i} T_{i 0} T_{i 1}=60 \\
& \text { (E-615) } 10080 \sum_{i}^{i} v_{i} \rho_{i}^{2} T_{i 0}^{2}=45 \\
& \text { (E-616) } 20160 \sum_{i} \sum_{j} v_{i} \tau_{i j}\left(T_{j 0}+2 T_{i 0}\right) T_{j 0}=18 \\
& \text { (E-617) } 6720 \sum_{i} v_{i} T_{i 0}^{3}=15 \\
& l=7: \quad(\mathrm{E}-701) \quad 72 \sum_{i} v_{i} \rho_{i}^{7}=1 \\
& \text { (E-702) } 3024 \sum_{i} v_{i} T_{i 5}=1 \\
& \text { (E-703) } \quad 15120 \sum_{i} v_{i} \rho_{i} T_{i 4}=7 \\
& \text { (E-704) } 30240 \sum_{i}^{i} v_{i} \rho_{i}^{2} T_{i 3}=21 \\
& \text { (E-705) } 60480 \sum_{i} \sum_{j} v_{i} \tau_{i j} T_{j 3}=1 \\
& \text { (E-706) } 30240 \sum_{i} v_{i} \rho_{i}^{3} T_{i 2}=35 \\
& (\mathrm{E}-707) \quad 181440 \sum_{i} \sum_{j} v_{i}\left(\rho_{i}+\rho_{j}\right) \tau_{i j} T_{j 2}=12 \\
& \text { (E-708) } 15120 \sum_{i} v_{i} \rho_{i}^{4} T_{i 1}=35 \\
& \text { (E-709) } 362880 \sum_{i} \sum_{j} v_{i} \rho_{i} \rho_{j} \tau_{i j} T_{j 1}=28 \\
& \text { (E-710) } 181440 \sum_{i} \sum_{j} v_{i}\left(\rho_{i}^{2}+\rho_{j}^{2}\right) \tau_{i j} T_{j 1}=31 \\
& \text { (E-711) } 362880 \sum_{i} \sum_{j} \sum_{k} v_{i} \tau_{i j} \tau_{j k} T_{k 1}=1
\end{aligned}
$$




$$
\begin{aligned}
& \text { (E-712) } 181440 \sum_{i} v_{i} T_{i 1} T_{i 2}=35 \\
& \text { (E-713) } 181440 \sum_{i} v_{i} \rho_{i} T_{i 1}^{2}=70 \\
& \text { (E-714) } 3024 \sum_{i} v_{i} \rho_{i}^{5} T_{i 0}=21 \\
& \text { (E-715) } 181440 \sum_{i} \sum_{j} v_{i} \rho_{i} \rho_{j}\left(\rho_{i}+\rho_{j}\right) \tau_{i j} T_{j 0}=105 \\
& \text { (E-716) } 60480 \sum_{i} \sum_{j} v_{i}\left(\rho_{i}^{3}+\rho_{j}^{3}\right) \tau_{i j} T_{j 0}=45 \\
& \text { (E-717) } 362880 \sum_{i} \sum_{j} \sum_{k} v_{i}\left(\rho_{i}+\rho_{i}+\rho_{k}\right) \tau_{i j} \tau_{j k} T_{k 0}=15 \\
& \text { (E-718) } 60480 \sum_{i} v_{i} T_{i 0} T_{i 3}=21 \\
& \text { (E-719) } 181440 \sum_{i} v_{i} \rho_{i} T_{i 0} T_{i 2}=105 \\
& \text { (E-720) } 181440 \sum_{i}^{i} v_{i} \rho_{i}^{2} T_{i 0} T_{i 1}=210 \\
& \text { (E-722) } 362880 \sum_{i} \sum_{j} v_{i} \tau_{i j}\left(T_{i 0} T_{j 1}+T_{j 0} T_{j 1}+T_{j 0} T_{i 1}\right)=66 \\
& \text { (E-723) } 30240 \sum_{i} v_{i} \rho_{i}^{3} T_{i 0}^{2}=105 \\
& \text { (E-724) } 181440 \sum_{i} \sum_{j} v_{i}\left(2 \rho_{i} T_{i 0}+\rho_{j} T_{j 0}\right) \tau_{i j} T_{j 0}=84 \\
& \text { (E-725) } 181440 \sum_{i} \sum_{j} v_{i}\left(\rho_{i} T_{j 0}+2 \rho_{j} T_{i 0}\right) \tau_{i j} T_{i 0}=120 \\
& \text { (E-726) } 181440 \sum_{i} v_{i} T_{i 0}^{2} T_{i 1}=105 \\
& \text { (E-727) } 60480 \sum_{i} v_{i} \rho_{i} T_{i 0}^{3}=105 \\
& l=8: \quad(\mathrm{E}-801) \quad 90 \sum_{i} v_{i} \rho_{i}^{8}=1 \\
& \text { (E-802) } 5040 \sum_{i} v_{i} T_{i 6}=1 \\
& \text { (E-803) } 30240 \sum_{i} v_{i} \rho_{i} T_{i 5}=8 \\
& \text { (E-804) } 75600 \sum_{i}^{i} v_{i} \rho_{i}^{2} T_{i 4}=28 \\
& \text { (E-805) } 151200 \sum_{i} \sum_{j} v_{i} \tau_{i j} T_{j 4}=1 \\
& \text { (E-806) } 100800 \sum_{i} v_{i} \rho_{i}^{3} T_{i 3}=56 \\
& \text { (E-807) } 604800 \sum_{i} \sum_{j} v_{i}\left(\rho_{i}+\rho_{j}\right) \tau_{i j} T_{j 3}=14 \\
& \text { (E-808) } 75600 \sum_{i} v_{i} \rho_{i}^{4} T_{i 2}=70 \\
& \text { (E-809) } 1814400 \sum_{i} \sum_{j} v_{i} \rho_{i} \rho_{j} \tau_{i j} T_{j 2}=40 \\
& \text { (E-810) } 907200 \sum_{i} \sum_{j} v_{i}\left(\rho_{i}^{2}+\rho_{j}^{2}\right) \tau_{i j} T_{j 2}=43 \\
& \text { (E-811) } 1814400 \sum_{i} \sum_{j} \sum_{k} v_{i} \tau_{i j} \tau_{j k} T_{k 2}=1 \\
& \text { (E-812) } 453600 \sum_{i} v_{i} T_{i 2}^{2}=35 \\
& \text { (E-813) } 30240 \sum_{i} v_{i} \rho_{i}^{5} T_{i 1}=56 \\
& \text { (E-814) } 1814400 \sum_{i} \sum_{j} v_{i} \rho_{i} \rho_{j}\left(\rho_{i}+\rho_{j}\right) \tau_{i j} T_{j 1}=192 \\
& \text { (E-815) } 604800 \sum_{i} \sum_{j} v_{i}\left(\rho_{i}^{3}+\rho_{j}^{3}\right) \tau_{i j} T_{j i}=76 \\
& \text { (E-816) } 3628800 \sum_{i} \sum_{j} \sum_{k} v_{i}\left(\rho_{i}+\rho_{j}+\rho_{k}\right) \tau_{i j} \tau_{j k} T_{k 1}=18
\end{aligned}
$$


(E-817) $604800 \sum_{i} v_{i} T_{i 1} T_{i 3}=56$

(E-818) $1814400 \sum_{i} v_{i} \rho_{i} T_{i 1} T_{i 2}=280$

(E-819) $907200 \sum_{i} v_{i} \rho_{i}^{2} T_{i 1}^{2}=280$

(E-820) $1814400 \sum_{i} \sum_{j} v_{i} \tau_{i j} T_{j 1}\left(2 T_{i 1}+T_{j 1}\right)=66$

(E-821) $5040 \sum_{i} v_{i} \rho_{i}^{6} T_{i 0}=28$

(E-822) $907200 \sum_{i} \sum_{j} v_{i} \rho_{i}^{2} \rho_{j}^{2} \tau_{i j} T_{j 0}=168$

(E-823) $604800 \sum_{i}^{i} \sum_{j}^{j} v_{i} \rho_{i} \rho_{j}\left(\rho_{i}^{2}+\rho_{j}^{2}\right) \tau_{i j} T_{j 0}=248$

(E-824) $151200 \sum_{i}^{i} \sum_{j}^{j} v_{i}\left(\rho_{i}^{4}+\rho_{j}^{4}\right) \tau_{i j} T_{j 0}=85$

(E-825) $3628800 \sum_{i} \sum_{j} \sum_{k} v_{i}\left(\rho_{i} \rho_{j}+\rho_{j} \rho_{k}+\rho_{j} \rho_{k}\right) \tau_{i j} \tau_{j k} T_{k 0}=82$

(E-826) $1814400 \sum_{i} \sum_{j} \sum_{k} v_{i}\left(\rho_{i}^{2}+\rho_{j}^{2}+\rho_{k}^{2}\right) \tau_{i j} \tau_{j k} T_{k 0}=49$

(E-827) $3628800 \sum_{i} \sum_{j} \sum_{k} \sum_{l} v_{i} \tau_{i j} \tau_{j k} \tau_{k l} T_{l 0}=1$

(E-828) $151200 \sum_{i} v_{i} T_{i 0} T_{i 4}=28$

(E-829) $604800 \sum_{i}^{i} v_{i} \rho_{i} T_{i 0} T_{i 3}=168$

(E-830) $907200 \sum_{i}^{i} v_{i} \rho_{i}^{2} T_{i 0} T_{i 2}=420$

(E-831) $1814400 \sum_{i} \sum_{j} v_{i} \tau_{i j}\left(T_{i 2} T_{j 0}+T_{i 0} T_{j 2}+T_{j 0} T_{j 2}\right)=113$

(E-832) $604800 \sum_{i} v_{i} \rho_{i}^{3} T_{i 0} T_{i 1}=560$

(E-833) $3628800 \sum_{i} \sum_{j} v_{i} \tau_{i j}\left(\rho_{j} T_{i 0} T_{j 1}+\rho_{j} T_{i 1} T_{j 0}+\rho_{i} T_{j 0} T_{j 1}\right)=360$

(E-834) $3628800 \sum_{i} \sum_{j} v_{i} \tau_{i j}\left(\rho_{i} T_{i 0} T_{j 1}+\rho_{i} T_{i 1} T_{j 0}+\rho_{j} T_{j 0} T_{j 1}\right)=508$

(E-835) $1814400 \sum_{i} v_{i} T_{i 0} T_{i 1}^{2}=280$

(E-836) $75600 \sum_{i} v_{i} \rho_{i}^{4} T_{i 0}^{2}=210$

(E-837) $907200 \sum_{i}^{i} \sum_{j} v_{i} \tau_{i j}\left(\rho_{i}^{2} T_{j 0}+2 \rho_{j}^{2} T_{i 0}\right) T_{j 0}=252$

(E-838) $1814400 \sum_{i} \sum_{j} v_{i} \rho_{i} \rho_{j} \tau_{i j}\left(2 T_{i 0}+T_{j 0}\right) T_{j 0}=624$

(E-839) $907200 \sum_{i} \sum_{j} v_{i} \tau_{i j}\left(\rho_{j}^{2} T_{j 0}+2 \rho_{i}^{2} T_{i 0}\right) T_{j 0}=465$

(E-840) $1814400 \sum_{i} \sum_{j} \sum_{k} v_{i} \tau_{i j}\left(2 \tau_{j k} T_{i 0} T_{k 0}+2 \tau_{j k} T_{j 0} T_{k 0}\right.$ $\left.+\tau_{i k} T_{j 0} T_{k 0}+\tau_{j k} T_{k 0}^{2}\right)=81$

(E-841) $907200 \sum_{i} v_{i} T_{i 0}^{2} T_{i 2}=210$

(E-842) $1814400 \sum_{i} v_{i} \rho_{i} T_{i 0}^{2} T_{i 1}=840$

(E-843) $302400 \sum_{i} v_{i} \rho_{i}^{2} T_{i 0}^{3}=420$

(E-844) $1814400 \sum_{i} \sum_{j} v_{i} \tau_{i j} T_{i 0} T_{j 0}^{2}=84$

(E-845) $604800 \sum_{i} \sum_{j} v_{i} \tau_{i j}\left(3 T_{i 0}^{2}+T_{j 0}^{2}\right) T_{j 0}=225$

(E-846) $151200 \sum_{i} v_{i} T_{i 0}^{4}=105$ 


\section{§1.5. Attainable Order of $(\mathbb{1}, \mathbb{1})$ - and $(\mathbb{1}, 2)-S t a g e$ Formulas}

The explicit $(1, q)$-stage formula is said to have the attainable order $m$ if $m$ is the largest integer for which

$$
y(x+h)-y_{1}=O\left(h^{m+1}\right)
$$

among all combinations of the parameters of the formula, where $y(x)$ is the analytical solution and $y_{1}$ is given by (1.3.6). The definition of the attainable order will be extended, if necessary, to general $(p, q)$-stage formula.

It is obvious that a $(1, q)$-stage formula has the attainable order $m$ if and only if the determining equations corresponding up to $l$ have at least one solution, but they have no solution up to $l+1$, where $l=m-2$.

Theorem 5. The attainable order of $(1,1)$-stage formula is 3 .

Proof. The left-hand side of the equation (E-22) is equal to

$$
24 \sum_{i} v_{i}\left(\sum_{j} \tau_{i j}\right)
$$

which vanishes for $(1,1)$-stage formula because $\sum_{j} \tau_{i j}=0$. This means that the parameters $v_{1}$ and $\rho_{1}$ can satisfy merely the equations (E-0) and (E-1).

Theorem 6. The attainable order of $(1,2)$-stage formula is equal to 4 .

Proof. Assume that the formula attains order 5, that is, the parameters $v_{1}, v_{2}, \rho_{1}, \rho_{2}$ and $\tau_{21}$ satisfy the equations (E-0)-(E-33).

$$
\begin{aligned}
& v_{1}+v_{2}=\frac{1}{2} \\
& v_{1} \rho_{1}+v_{2} \rho_{2}=\frac{1}{6} \\
& v_{2} \tau_{21}=\frac{1}{24} \\
& v_{1} \rho_{1}^{2}+v_{2} \rho_{2}^{2}=\frac{1}{12} \\
& v_{2} \rho_{2} \tau_{21}=\frac{1}{40} \\
& v_{2} \rho_{1} \tau_{21}=\frac{1}{120}
\end{aligned}
$$




$$
v_{1} \rho_{1}^{3}+v_{2} \rho_{2}^{3}=\frac{1}{20}
$$

The equations (1), (2) and (4) yield a matrix equation

$$
\left(\begin{array}{ccc}
1 & 1 & 1 / 2 \\
\rho_{1} & \rho_{2} & 1 / 6 \\
\rho_{1}^{2} & \rho_{2}^{2} & 1 / 12
\end{array}\right)\left(\begin{array}{c}
v_{1} \\
v_{2} \\
-1
\end{array}\right)=0
$$

which implies

$$
\left|\begin{array}{ccc}
1 & 1 & 1 / 2 \\
\rho_{1} & \rho_{2} & 1 / 6 \\
\rho_{1}^{2} & \rho_{2}^{2} & 1 / 12
\end{array}\right|=0
$$

for the existence of nontrivial solution $\left[v_{1}, v_{2},-1\right]^{t}$. On the other hand, (3), (5) and (6) give the values

$$
\rho_{1}=\frac{1}{5}, \quad \rho_{2}=\frac{3}{5}
$$

which specify the determinant of (8) by $1 / 250$. This contradiction implies the statement.

Note. SHINTANI gives $(1,1)$-stage formula with parameters $v_{1}=1 / 2$ and $\rho_{1}=1 / 3$. He also gives $(1,2)$-stage formula with $v_{1}=(9+\sqrt{6}) / 36, v_{2}=(9-$ $\sqrt{6}) / 36, \rho_{1}=(4-\sqrt{6}) / 10, \rho_{2}=(4+\sqrt{6}) / 10, \tau_{21}=(9+\sqrt{6}) / 50$. These parameters are not unique solution (1.5.1)-(1.5.4). The solution of them is represented with one parameter $\rho$ by the following:

$$
\begin{aligned}
& \rho_{1}=\rho \quad\left(\rho \neq \frac{1}{3}\right) \\
& \rho_{2}=(2 \rho-1) / 2(3 \rho-1), \\
& v_{1}=1 / 6\left(6 \rho^{2}-4 \rho+1\right), \\
& v_{2}=(3 \rho-1)^{2} / 3\left(6 \rho^{2}-4 \rho+1\right) \\
& \tau_{21}=\left(6 \rho^{2}-4 \rho+1\right) / 8(3 \rho-1)^{2}
\end{aligned}
$$

\section{§1.6. Attainable Order of $(1,3)$-Stage Formula}

The determining equation for the explicit $(1,3)$-stage formula are given as follows : 


$$
\begin{aligned}
& v_{1}+v_{2}+v_{3}=\frac{1}{2} \\
& v_{1} \rho_{1}+v_{2} \rho_{2}+v_{3} \rho_{3}=\frac{1}{6} \\
& v_{1} \rho_{1}^{2}+v_{2} \rho_{2}^{2}+v_{3} \rho_{3}^{2}=\frac{1}{12}
\end{aligned}
$$

$$
v_{2} \tau_{21}+v_{3}\left(\tau_{31}+\tau_{32}\right)=\frac{1}{24}
$$

$$
v_{1} \rho_{1}^{3}+v_{2} \rho_{2}^{3}+v_{3} \rho_{3}^{3}=\frac{1}{20}
$$

$$
v_{2} \rho_{2} \tau_{21}+v_{3} \rho_{3}\left(\tau_{31}+\tau_{32}\right)=\frac{1}{40}
$$

$$
v_{2} \rho_{1} \tau_{21}+v_{3}\left(\rho_{1} \tau_{31}+\rho_{2} \tau_{32}\right)=\frac{1}{120}
$$$$
v_{1} \rho_{1}^{4}+v_{2} \rho_{2}^{4}+v_{3} \rho_{3}^{4}=\frac{1}{30}
$$

$$
v_{2} \rho_{2}^{2} \tau_{21}+v_{3} \rho_{3}^{2}\left(\tau_{31}+\tau_{32}\right)=\frac{1}{60}
$$

$$
v_{2} \rho_{2} \rho_{1} \tau_{21}+v_{3} \rho_{3}\left(\rho_{1} \tau_{31}+\rho_{2} \tau_{32}\right)=\frac{1}{180}
$$

$$
v_{2} \rho_{1}^{2} \tau_{21}+v_{3}\left(\rho_{1}^{2} \tau_{31}+\rho_{2}^{2} \tau_{32}\right)=\frac{1}{360}
$$

$$
v_{2} \tau_{21}^{2}+v_{3}\left(\tau_{31}+\tau_{32}\right)^{2}=\frac{1}{120}
$$

$$
v_{3} \tau_{21} \tau_{32}=\frac{1}{720}
$$

It is remarkable that none of the factors on the left of (E-46) can vanish.

Assume that two of $\rho_{1}, \rho_{2}, \rho_{3}$ are equal, say $\rho_{I}=\rho_{J}$. Then, from (E-0), $(E-1),(E-21),(E-31)$ and $(E-41)$, we see that

$$
\begin{aligned}
& {\left[\begin{array}{lll}
1 & 1 & \frac{1}{2} \\
\rho_{I} & \rho_{K} & \frac{1}{6} \\
\rho_{I}^{2} & \rho_{K}^{2} & \frac{1}{12}
\end{array}\right]\left[\begin{array}{c}
v_{I}+v_{J} \\
v_{K} \\
-1
\end{array}\right]=0,} \\
& {\left[\begin{array}{ccc}
1 & 1 & \frac{1}{6} \\
\rho_{I} & \rho_{K} & \frac{1}{12} \\
\rho_{I}^{2} & \rho_{K}^{2} & \frac{1}{20}
\end{array}\right]\left[\begin{array}{c}
\rho_{I}\left(v_{I}+v_{J}\right) \\
\rho_{K} v_{K} \\
-1
\end{array}\right]=0,}
\end{aligned}
$$




$$
\left[\begin{array}{ccc}
1 & 1 & \frac{1}{12} \\
\rho_{I} & \rho_{K} & \frac{1}{20} \\
\rho_{I}^{2} & \rho_{K}^{2} & \frac{1}{30}
\end{array}\right]\left[\begin{array}{c}
\rho_{I}^{2}\left(v_{I}+v_{J}\right) \\
\rho_{K}^{2} v_{K} \\
-1
\end{array}\right]=0
$$

The condition that the above three equations have non-trivial solutions, implies the equations with respect to $\rho_{I}, \rho_{K}$ as follows:

$$
\begin{aligned}
& \left(\rho_{K}-\rho_{I}\right)\left\{\frac{1}{2} \rho_{I} \rho_{K}-\frac{1}{6}\left(\rho_{I}+\rho_{K}\right)+\frac{1}{12}\right\}=0, \\
& \left(\rho_{K}-\rho_{I}\right)\left\{\frac{1}{6} \rho_{I} \rho_{K}-\frac{1}{12}\left(\rho_{I}+\rho_{K}\right)+\frac{1}{20}\right\}=0
\end{aligned}
$$

and

$$
\left(\rho_{K}-\rho_{I}\right)\left\{\frac{1}{12} \rho_{I} \rho_{K}-\frac{1}{20}\left(\rho_{I}+\rho_{K}\right)+\frac{1}{30}\right\}=0 .
$$

If $\rho_{I} \neq \rho_{K}, \rho_{I}$ and $\rho_{K}$ must satisfy the equations

$$
\begin{aligned}
\frac{1}{2} \rho_{I} \rho_{K}-\frac{1}{6}\left(\rho_{I}+\rho_{K}\right)+\frac{1}{12} & =\frac{1}{6} \rho_{I} \rho_{K}-\frac{1}{12}\left(\rho_{I}+\rho_{K}\right)+\frac{1}{20} \\
& =\frac{1}{12} \rho_{I} \rho_{K}-\frac{1}{20}\left(\rho_{I}+\rho_{K}\right)+\frac{1}{30}=0,
\end{aligned}
$$

which is impossible. The case of $\rho_{1}=\rho_{2}=\rho_{3}$ leads to a contradiction because of

$$
\begin{aligned}
& v_{1}+v_{2}+v_{3}=\frac{1}{2}, \\
& \rho_{1}\left(v_{1}+v_{2}+v_{3}\right)=\frac{1}{6}, \\
& \rho_{1}^{2}\left(v_{1}+v_{2}+v_{3}\right)=\frac{1}{12},
\end{aligned}
$$

induced by $(\mathrm{E}-0),(\mathrm{E}-1),(\mathrm{E}-21)$, respectively.

Hence no two of $\rho_{1}, \rho_{2}, \rho_{3}$ are equal. It is convenient to define

$$
A_{i}=\sum_{j=1}^{i-1} \tau_{i j}-\frac{1}{2} \rho_{i}^{2} \quad(i=1,2,3) .
$$

Since we consider the explicit formula, $A_{1}=-\rho_{1}^{2} / 2$ holds. Then, we have simultaneous linear equations

$$
\left[\begin{array}{ccc}
1 & 1 & 1 \\
\rho_{1} & \rho_{2} & \rho_{3} \\
\rho_{1}^{2} & \rho_{2}^{2} & \rho_{3}^{2}
\end{array}\right]\left[\begin{array}{l}
v_{1} A_{1} \\
v_{2} A_{2} \\
v_{3} A_{3}
\end{array}\right]=0
$$


by (E-21), (E-22), (E-31), (E-32), (E-41) and (E-42). Since no two of $\rho_{1}, \rho_{2}$, $\rho_{3}$ are equal, all of $v_{1} A_{1}, v_{2} A_{2}, v_{3} A_{3}$ vanish. By virtue of the above mentioned remark, we distinguish the following four cases.

Case 1. $A_{1}=A_{2}=A_{3}=0$.

The equations $A_{1}=0$ and $A_{2}=0$ imply $\rho_{1}=0$ and $\tau_{21}=\rho_{2}^{2} / 2$. Then, (E-43) and (E-44) bring the equations $v_{3} \rho_{2} \rho_{3} \tau_{32}=1 / 180$ and $v_{3} \rho_{2}^{2} \tau_{32}=1 / 360$, which give the identity $\rho_{3}=2 \rho_{2}$. Thus $(\mathrm{E}-1),(\mathrm{E}-21)$ and $(\mathrm{E}-31)$ imply the equation

$$
\left[\begin{array}{ccc}
\rho_{2} & 2 \rho_{2} & \frac{1}{6} \\
\rho_{2}^{2} & 4 \rho_{2}^{2} & \frac{1}{12} \\
\rho_{3}^{3} & 8 \rho_{3}^{3} & \frac{1}{20}
\end{array}\right]\left[\begin{array}{c}
v_{2} \\
v_{3} \\
-1
\end{array}\right]=0
$$

which yields a quadratic equation of $\rho_{2}$

$$
20 \rho_{2}^{2}-15 \rho_{2}+3=0
$$

because of $\rho_{2} \neq 0$. But the above quadratic equation has no real roots.

Case 2. $v_{1}=0$ and $A_{2}=A_{3}=0$.

In such case, we have the equations

$$
\tau_{21}=\frac{1}{2} \rho_{2}^{2}
$$

and

$$
\tau_{31}+\tau_{32}=\frac{1}{2} \rho_{3}^{2}
$$

Substitution of $\tau_{21}=\rho_{2}^{2} / 2$ and $\tau_{31}=\left(\rho_{3}^{2} / 2\right)-\tau_{32}$ into (E-44) implies

$$
\rho_{1}^{2}+24 v_{3} \tau_{32}\left(\rho_{2}^{2}-\rho_{1}^{2}\right)=\frac{1}{15} \text {. }
$$

Employing the equation

$$
v_{3} \rho_{2}^{2} \tau_{32}=\frac{1}{360}
$$

induced by (E-46), we obtain

$$
\rho_{1}\left(1-24 v_{3} \tau_{32}\right)=0 .
$$

Hence, the equation $\rho_{1}=0$ or $v_{3} \tau_{32}=1 / 24$ holds. The case of $\rho_{1}=0$ is equivalent to Case 1 . The equation $v_{3} \tau_{32}=1 / 24$ yields 


$$
\rho_{1}+24 v_{3} \tau_{32}\left(\rho_{2}-\rho_{1}\right)=\rho_{2}=\frac{1}{5}
$$

by (E-33). The equation (*), however, implies $\rho_{2}^{2}=1 / 15$, which leads to a contradiction.

Case 3. $v_{2}=0$ and $A_{1}=A_{3}=0$.

$A_{1}=0$ implies $\rho_{1}=0$. Then, $(\mathrm{E}-1)$ and (E-21) bring the equations

$$
v_{3} \rho_{3}=\frac{1}{6} .
$$

and

$$
v_{3} \rho_{3}^{2}=\frac{1}{12}
$$

Hence we see $\rho_{3}=1 / 2$ and $v_{3}=1 / 3$. It contradicts the equation $v_{3} \rho_{3}^{3}=1 / 20$ induced by $(\mathrm{E}-31)$.

Case 4. $v_{1}=0, v_{2}=0$ and $A_{3}=0$.

The equations (E-0) and (E-1) yield $v_{3}=1 / 2$ and $\rho_{3}=1 / 3$. Again, it contradicts the equation $v_{3} \rho_{3}^{3}=1 / 20$ induced by $(\mathrm{E}-31)$.

Thus, we can conclude that the determining equations $(E-0)-(E-46)$ have no solutions. The proof of the following theorem is now accomplished.

Theorem 7. The explicit (1, 3)-stage formula can not attain order 6. Its attainable order is 5 .

Note. Shintani gives $(1,3)$-stage formula with parameters

$$
\begin{aligned}
v_{1} & =1 / 12, v_{2}=(5+\sqrt{5}) / 24, v_{3}=(5-\sqrt{5}) / 24, \rho_{1}=0, \rho_{2}=(5-\sqrt{5}) / 10, \\
\tau_{21} & =(3-\sqrt{5}) / 20, \rho_{3}=(5+\sqrt{5}) / 10, \tau_{31}=0 \text { and } \tau_{32}=(3+\sqrt{5}) / 20
\end{aligned}
$$

These parameters are also not unique solution of $(E-0)-(E-33)$.

\section{§ 1.7. Attainable Order of $(1,4)$-Stage Formula}

The determining equation for the explicit $(1,4)$-stage formula are given by the following:

(E-0) $v_{1}+v_{2}+v_{3}+v_{4}=\frac{1}{2}$
(E-1) $\quad v_{1} \rho_{1}+v_{2} \rho_{2}+v_{3} \rho_{3}+v_{4} \rho_{4}=\frac{1}{6}$ 
(E-21) $v_{1} \rho_{1}^{2}+v_{2} \rho_{2}^{2}+v_{3} \rho_{3}^{2}+v_{4} \rho_{4}^{2}=\frac{1}{12}$

$(\mathrm{E}-22) \quad v_{2} \tau_{21}+v_{3}\left(\tau_{31}+\tau_{32}\right)+v_{4}\left(\tau_{41}+\tau_{42}+\tau_{43}\right)=\frac{1}{24}$

(E-31) $\quad v_{1} \rho_{1}^{3}+v_{2} \rho_{2}^{3}+v_{3} \rho_{3}^{3}+v_{4} \rho_{4}^{3}=\frac{1}{20}$

(E-32) $v_{2} \rho_{2} \tau_{21}+v_{3} \rho_{3}\left(\tau_{31}+\tau_{32}\right)+v_{4} \rho_{4}\left(\tau_{41}+\tau_{42}+\tau_{43}\right)=\frac{1}{40}$

(E-33) $v_{2} \rho_{1} \tau_{21}+v_{3}\left(\rho_{1} \tau_{31}+\rho_{2} \tau_{32}\right)+v_{4}\left(\rho_{1} \tau_{41}+\rho_{2} \tau_{42}+\rho_{3} \tau_{43}\right)=\frac{1}{120}$

$(\mathrm{E}-41) \quad v_{1} \rho_{1}^{4}+v_{2} \rho_{2}^{4}+v_{3} \rho_{3}^{4}+v_{4} \rho_{4}^{4}=\frac{1}{30}$

$(\mathrm{E}-42) \quad v_{2} \rho_{2}^{2} \tau_{21}+v_{3} \rho_{3}^{2}\left(\tau_{31}+\tau_{32}\right)+v_{4} \rho_{4}^{2}\left(\tau_{41}+\tau_{42}+\tau_{43}\right)=\frac{1}{60}$

(E-43) $v_{2} \rho_{2} \rho_{1} \tau_{21}+v_{3} \rho_{3}\left(\rho_{1} \tau_{31}+\rho_{2} \tau_{32}\right)+v_{4} \rho_{4}\left(\rho_{1} \tau_{41}+\rho_{2} \tau_{42}+\rho_{3} \tau_{43}\right)=\frac{1}{180}$

(E-44) $v_{2} \rho_{1}^{2} \tau_{21}+v_{3}\left(\rho_{1}^{2} \tau_{31}+\rho_{2}^{2} \tau_{32}\right)+v_{4}\left(\rho_{1}^{2} \tau_{41}+\rho_{2}^{2} \tau_{42}+\rho_{3}^{2} \tau_{43}\right)=\frac{1}{360}$

$(\mathrm{E}-45) \quad v_{3} \tau_{21} \tau_{32}+v_{4}\left(\tau_{21} \tau_{42}+\tau_{31} \tau_{43}+\tau_{32} \tau_{43}\right)=\frac{1}{720}$

$(\mathrm{E}-46) \quad v_{2} \tau_{21}^{2}+v_{3}\left(\tau_{31}+\tau_{32}\right)^{2}+v_{4}\left(\tau_{41}+\tau_{42}+\tau_{43}\right)^{2}=\frac{1}{120}$

(E-51) $v_{1} \rho_{1}^{5}+v_{2} \rho_{2}^{5}+v_{3} \rho_{3}^{5}+v_{4} \rho_{4}^{5}=\frac{1}{42}$

$(\mathrm{E}-52) \quad v_{2} \rho_{2}^{3} \tau_{21}+v_{3} \rho_{3}^{3}\left(\tau_{31}+\tau_{32}\right)+v_{4} \rho_{4}^{3}\left(\tau_{41}+\tau_{42}+\tau_{43}\right)=\frac{1}{84}$

(E-53) $\quad v_{2} \rho_{2}^{2} \rho_{1} \tau_{21}+v_{3} \rho_{3}^{2}\left(\rho_{1} \tau_{31}+\rho_{2} \tau_{32}\right)+v_{4} \rho_{4}^{2}\left(\rho_{1} \tau_{41}+\rho_{2} \tau_{42}+\rho_{3} \tau_{43}\right)=\frac{1}{252}$

(E-54) $v_{2} \rho_{2} \rho_{1}^{2} \tau_{21}+v_{3} \rho_{3}\left(\rho_{1}^{2} \tau_{31}+\rho_{2}^{2} \tau_{32}\right)+v_{4} \rho_{4}\left(\rho_{1}^{2} \tau_{41}+\rho_{2}^{2} \tau_{42}+\rho_{3}^{2} \tau_{43}\right)=\frac{1}{504}$

(E-55) $\quad v_{2} \rho_{1}^{3} \tau_{21}+v_{3}\left(\rho_{1}^{3} \tau_{31}+\rho_{2}^{3} \tau_{32}\right)+v_{4}\left(\rho_{1}^{3} \tau_{41}+\rho_{2}^{3} \tau_{42}+\rho_{3}^{3} \tau_{43}\right)=\frac{1}{840}$

(E-56) $\quad v_{3}\left(\rho_{2}+\rho_{3}\right) \tau_{21} \tau_{32}+v_{4}\left(\rho_{2}+\rho_{4}\right) \tau_{21} \tau_{42}+v_{4}\left(\rho_{3}+\rho_{4}\right)\left(\tau_{31} \tau_{43}+\tau_{32} \tau_{43}\right)=\frac{1}{630}$

(E-57) $\quad v_{3} \rho_{1} \tau_{21} \tau_{32}+v_{4} \rho_{1}\left(\tau_{21} \tau_{42}+\tau_{31} \tau_{43}\right)+v_{4} \rho_{2} \tau_{32} \tau_{43}=\frac{1}{5040}$

(E-58) $\quad v_{2} \rho_{1} \tau_{21}^{2}+v_{3}\left(\rho_{1} \tau_{31}^{2}+\rho_{1} \tau_{31} \tau_{32}+\rho_{2} \tau_{31} \tau_{32}+\rho_{2} \tau_{32}^{2}\right)$

$$
\begin{aligned}
& +v_{4}\left(\rho_{1} \tau_{41}^{2}+\rho_{2} \tau_{42}^{2}+\rho_{3} \tau_{43}^{2}\right)+v_{4} \rho_{1}\left(\tau_{41} \tau_{42}+\tau_{41} \tau_{43}\right) \\
& +v_{4} \rho_{2}\left(\tau_{41} \tau_{42}+\tau_{42} \tau_{43}\right)+v_{4} \rho_{3}\left(\tau_{31} \tau_{43}+\tau_{42} \tau_{43}\right)=\frac{1}{504}
\end{aligned}
$$

(E-59) $v_{2} \rho_{2} \tau_{21}^{2}+v_{3} \rho_{3}\left(\tau_{31}+\tau_{32}\right)^{2}+v_{4} \rho_{4}\left(\tau_{41}+\tau_{42}+\tau_{43}\right)^{2}=\frac{1}{168}$ 
The question is whether any parameters $v_{i}, \rho_{i}$ and $\tau_{i j}$ exist to satisfy these 22 equations simultaneously. It is helpful for investigation to introduce the following notations:

$$
T_{i 0}=\sum_{j=1}^{i-1} \tau_{i j}, \quad T_{i 1}=\sum_{j=1}^{i-1} \rho_{j} \tau_{i j} \quad(i=1,2,3,4),
$$

( $T_{10}$ and $T_{11}$ mean zero.)

$$
A_{i}=T_{i 0}-\frac{1}{2} \rho_{i}^{2}, \quad B_{i}=T_{i 1}-\frac{1}{6} \rho_{i}^{3} \quad(i=1,2,3,4) .
$$

Then, from (E-21), (E-22), (E-31), (E-32), (E-33), (E-41), (E-42), (E-43), (E-51), (E-52) and (E-53), we easily see that

$$
\sum_{i} v_{i} A_{i}=\sum_{i} v_{i} \rho_{i} A_{i}=\sum_{i} v_{i} \rho_{i}^{2} A_{i}=\sum_{i} v_{i} \rho_{i}^{3} A_{i}=0
$$

and

$$
\sum_{i} v_{i} B_{i}=\sum_{i} v_{i} \rho_{i} B_{i}=\sum_{i} v_{i} \rho_{i}^{2} B_{i}=0
$$

The equations (1) means

$$
\left(\begin{array}{cccc}
1 & 1 & 1 & 1 \\
\rho_{1} & \rho_{2} & \rho_{3} & \rho_{4} \\
\rho_{1}^{2} & \rho_{2}^{2} & \rho_{3}^{2} & \rho_{4}^{2} \\
\rho_{1}^{3} & \rho_{2}^{3} & \rho_{3}^{3} & \rho_{4}^{3}
\end{array}\right)\left(\begin{array}{l}
v_{1} A_{1} \\
v_{2} A_{2} \\
v_{3} A_{3} \\
v_{4} A_{4}
\end{array}\right)=0
$$

We now distinguish two cases according as two of $\rho_{1}, \rho_{2}, \rho_{3}, \rho_{4}$ are equal or otherwise.

Case 1. Two of $\rho_{1}, \rho_{2}, \rho_{3}, \rho_{4}$ are equal.

Assume that two of them are equal, say $\rho_{I}=\rho_{J}$. Equations (E-0), (E-1), (E-21), (E-31), (E-41) and (E-51) give three simultaneous linear equations as follows:

$$
\left(\begin{array}{cccc}
1 & 1 & 1 & \frac{1}{2} \\
\rho_{I} & \rho_{K} & \rho_{L} & \frac{1}{6} \\
\rho_{I}^{2} & \rho_{K}^{2} & \rho_{L}^{2} & \frac{1}{12} \\
\rho_{I}^{3} & \rho_{K}^{3} & \rho_{L}^{3} & \frac{1}{20}
\end{array}\right)\left(\begin{array}{c}
v_{I}+v_{J} \\
v_{K} \\
v_{L} \\
-1
\end{array}\right)=0
$$




$$
\begin{gathered}
\left(\begin{array}{cccc}
1 & 1 & 1 & \frac{1}{6} \\
\rho_{I} & \rho_{K} & \rho_{L} & \frac{1}{12} \\
\rho_{I}^{2} & \rho_{K}^{2} & \rho_{L}^{2} & \frac{1}{20} \\
\rho_{I}^{3} & \rho_{K}^{3} & \rho_{L}^{3} & \frac{1}{30}
\end{array}\right)\left(\begin{array}{c}
\rho_{I}\left(v_{I}+v_{K}\right) \\
\rho_{K} v_{K} \\
\rho_{L} v_{K} \\
-1
\end{array}\right)=0, \\
\left(\begin{array}{cccc}
1 & 1 & 1 & \frac{1}{12} \\
\rho_{I} & \rho_{K} & \rho_{L} & \frac{1}{20} \\
\rho_{I}^{2} & \rho_{K}^{2} & \rho_{L}^{2} & \frac{1}{30} \\
\rho_{I}^{3} & \rho_{K}^{3} & \rho_{L}^{3} & \frac{1}{42}
\end{array}\right)\left(\begin{array}{c}
\rho_{I}^{2}\left(v_{I}+v_{J}\right) \\
\rho_{K}^{2} v_{K} \\
\rho_{L}^{2} v_{L} \\
-1
\end{array}\right)=0 .
\end{gathered}
$$

The condition that these equations have non-trivial solutions, implies the determinants of matrices to be vanishing. Hence, we see that the equations

$$
\begin{aligned}
\frac{1}{60}\left(\rho_{I}-\rho_{K}\right)\left(\rho_{K}-\rho_{L}\right)\left(\rho_{L}-\rho_{I}\right)\{ & -30 \rho_{I} \rho_{K} \rho_{L}+10\left(\rho_{I} \rho_{K}+\rho_{K} \rho_{L}+\rho_{L} \rho_{I}\right) \\
& \left.-5\left(\rho_{I}+\rho_{K}+\rho_{L}\right)+3\right\}=0 \\
\frac{1}{60}\left(\rho_{I}-\rho_{K}\right)\left(\rho_{K}-\rho_{L}\right)\left(\rho_{L}-\rho_{I}\right)\{ & -10 \rho_{I} \rho_{K} \rho_{L}+5\left(\rho_{I} \rho_{K}+\rho_{K} \rho_{L}+\rho_{L} \rho_{I}\right) \\
- & \left.-3\left(\rho_{I}+\rho_{K}+\rho_{L}\right)+2\right\}=0
\end{aligned}
$$

and

$$
\begin{aligned}
\frac{1}{420}\left(\rho_{I}-\rho_{K}\right)\left(\rho_{K}-\rho_{L}\right)\left(\rho_{L}-\rho_{I}\right)\{ & -35 \rho_{I} \rho_{K} \rho_{L}+21\left(\rho_{I} \rho_{K}+\rho_{K} \rho_{L}+\rho_{L} \rho_{I}\right) \\
- & \left.14\left(\rho_{I}+\rho_{K}+\rho_{L}\right)+10\right\}=0
\end{aligned}
$$

hold. We can distinguish three cases.

(i) At least three of $\rho_{i}$ are equal.

(ii) $\rho_{I}=\rho_{J}$ and $\rho_{K}=\rho_{L}$.

(iii) $\rho_{I}, \rho_{K}, \rho_{L}$ are distinct, and the above equations hold.

But, the case (i) can not hold by the similar reason mentioned at the first part of Section 1.6. In the case (ii), $(\mathrm{E}-0),(\mathrm{E}-1),(\mathrm{E}-21),(\mathrm{E}-31)$ and $(\mathrm{E}-41)$ imply the equations 


$$
\begin{aligned}
& \left(\begin{array}{ccc}
1 & 1 & \frac{1}{2} \\
\rho_{I} & \rho_{K} & \frac{1}{6} \\
\rho_{I}^{2} & \rho_{K}^{2} & \frac{1}{12}
\end{array}\right)\left(\begin{array}{c}
v_{I}+v_{J} \\
v_{K}+v_{L} \\
-1
\end{array}\right)=0, \\
& \left(\begin{array}{ccc}
1 & 1 & \frac{1}{6} \\
\rho_{I} & \rho_{K} & \frac{1}{12} \\
\rho_{I}^{2} & \rho_{K}^{2} & \frac{1}{20}
\end{array}\right)\left(\begin{array}{c}
\rho_{I}\left(v_{I}+v_{J}\right) \\
\rho_{K}\left(v_{K}+v_{L}\right) \\
-1
\end{array}\right)=0, \\
& \left(\begin{array}{ccc}
1 & 1 & \frac{1}{12} \\
\rho_{I} & \rho_{K} & \frac{1}{20} \\
\rho_{I}^{2} & \rho_{K}^{2} & \frac{1}{30}
\end{array}\right)\left(\begin{array}{c}
\rho_{I}^{2}\left(v_{I}+v_{J}\right) \\
\rho_{K}^{2}\left(v_{K}+v_{L}\right) \\
-1
\end{array}\right)=0 .
\end{aligned}
$$

Thus, by the same reason as for case (i), these equations lead to a contradiction. In the case (iii), we easily see the equations

$$
\begin{gathered}
\rho_{I}+\rho_{K}+\rho_{L}=\frac{9}{7}, \\
\rho_{I} \rho_{K}+\rho_{K} \rho_{L}+\rho_{L} \rho_{I}=\frac{3}{7}
\end{gathered}
$$

and

$$
\rho_{1} \rho_{K} \rho_{L}=\frac{1}{35}
$$

which imply that $\rho_{I}, \rho_{K}, \rho_{L}$ are distinct roots of the cubic equation

$$
x^{3}-\frac{9}{7} x^{2}+\frac{3}{7} x-\frac{1}{35}=0 .
$$

The cubic equation is irreducible and has three distinct real roots given as follows. Let $\theta$ be an angle such that

$$
\cos 3 \theta=\frac{1}{5 \sqrt{2}} \quad\left(0<3 \theta \leqq \frac{\pi}{2}\right) .
$$

Then, the roots are 


$$
\left\{\begin{array}{l}
R_{0}=\frac{1}{7}(3+2 \sqrt{2} \cos \theta) \\
R_{1}=\frac{1}{7}(3-\sqrt{2} \cos \theta+\sqrt{6} \sin \theta) \\
R_{-1}=\frac{1}{7}(3-\sqrt{2} \cos \theta-\sqrt{6} \sin \theta)
\end{array}\right.
$$

Some algebraic properties on the equation (3) are the followings:

Lemma 1.7.1. The roots $R_{0}, R_{1}, R_{-1}$ are equal to none of $0,1 / 7,1 / 3$.

Proof. Substitution of $0,1 / 7$ and $1 / 3$ into the cubic polynomial of (3) gives $-1 / 35,16 / 1715$ and $8 / 945$, respectively.

Lemma 1.7.2. The equation (3) has no common roots with the cubic equation

$$
x^{3}-\frac{3}{8} x^{2}-\frac{3}{16} x+\frac{3}{64}=0
$$

Proof. Put

$$
f_{1}(x)=x^{3}-\frac{9}{7} x^{2}+\frac{3}{7} x-\frac{1}{35}
$$

and

$$
f_{2}(x)=x^{3}-\frac{3}{8} x^{2}-\frac{3}{16} x+\frac{3}{64}
$$

The Sylvester's determinant $D\left(f_{1}, f_{2}\right)$ is equal to $-3437 / 32768000$.

Lemma 1.7.3. The equation (3) has no roots, one of which is the triple of another.

Proof. Assume that one of roots is equal to the triple of another, then the root satisfy another cubic equation

$$
27 x^{3}-\frac{81}{7} x^{2}+\frac{9}{7} x-\frac{1}{35}=0 .
$$

That is,

$$
x^{3}-\frac{3}{7} x^{2}+\frac{1}{21} x-\frac{1}{945}=0
$$

Put

$$
f_{1}(x)=x^{3}-\frac{9}{7} x^{2}+\frac{3}{7} x-\frac{1}{35}
$$

and 


$$
f_{2}(x)=x^{3}-\frac{7}{3} x^{2}+\frac{1}{21} x-\frac{1}{945}
$$

The Sylvester's determinant $D\left(f_{1}, f_{2}\right)$ is equal to $80384 / 41351522625$.

Case 1.1. $\rho_{1}=\rho_{2}$.

From (E-45) and (E-57), the assumption gives $\rho_{1}=\rho_{2}=1 / 7$. Due to Lemma 1.7.1, we lead to a contradiction.

Case 1.2. $\rho_{1}=\rho_{3}$.

We may assume that $\rho_{1}, \rho_{2}, \rho_{4}$ are distinct. From $(\mathrm{E}-0),(\mathrm{E}-1)$ and (E-21), we have the equation

$$
\left(\begin{array}{ccc}
1 & 1 & 1 \\
\rho_{1} & \rho_{2} & \rho_{4} \\
\rho_{1}^{2} & \rho_{2}^{2} & \rho_{4}^{2}
\end{array}\right)\left(\begin{array}{c}
v_{1}+v_{3} \\
v_{2} \\
v_{4}
\end{array}\right)=\left(\begin{array}{c}
\frac{1}{2} \\
\frac{1}{6} \\
\frac{1}{12}
\end{array}\right)
$$

The solution of (9) is given by

$$
\begin{aligned}
& \left(\begin{array}{c}
v_{1}+v_{3} \\
v_{2} \\
v_{4}
\end{array}\right)=\left(\begin{array}{ccc}
1 & 1 & 1 \\
\rho_{1} & \rho_{2} & \rho_{4} \\
\rho_{1}^{2} & \rho_{2}^{2} & \rho_{4}^{2}
\end{array}\right)^{-1}\left(\begin{array}{c}
\frac{1}{2} \\
\frac{1}{6} \\
\frac{1}{12}
\end{array}\right) \\
& =\left(\begin{array}{l}
\frac{-6 \rho_{2} \rho_{4}+2\left(\rho_{2}+\rho_{4}\right)-1}{12\left(\rho_{1}-\rho_{2}\right)\left(\rho_{4}-\rho_{1}\right)} \\
\frac{-6 \rho_{4} \rho_{1}+2\left(\rho_{4}+\rho_{1}\right)-1}{12\left(\rho_{1}-\rho_{2}\right)\left(\rho_{2}-\rho_{4}\right)} \\
\frac{-6 \rho_{1} \rho_{2}+2\left(\rho_{1}+\rho_{2}\right)-1}{12\left(\rho_{2}-\rho_{4}\right)\left(\rho_{4}-\rho_{1}\right)}
\end{array}\right)
\end{aligned}
$$

Note that $v_{1}+v_{3}, v_{2}, v_{4}$ can not vanish. The reason is as follows: For example, assume that $-6 \rho_{2} \rho_{4}+2\left(\rho_{2}+\rho_{4}\right)-1=0$. Then

$$
\rho_{4}=\frac{2 \rho_{2}-1}{6 \rho_{2}-2}
$$

holds. Substituting this into the cubic equation

$$
\rho_{4}^{3}-\frac{9}{7} \rho_{4}^{2}+\frac{3}{7} \rho_{4}-\frac{1}{35}=0,
$$

we see that 


$$
\frac{64 \rho_{2}^{3}-24 \rho_{2}^{2}-12 \rho_{2}+3}{35\left(6 \rho_{2}-2\right)^{3}}=0 .
$$

But, by Lemma 1.7.2, there is no common roots for the cubic equations

$$
\rho_{2}^{3}-\frac{9}{7} \rho_{2}^{2}+\frac{3}{7} \rho_{2}-\frac{1}{35}=0
$$

and

$$
\rho_{2}^{3}-\frac{3}{8} \rho_{2}^{2}-\frac{3}{16} \rho_{2}+\frac{3}{64}=0
$$

Then, from the equations (1) and (2) we have

$$
v_{1} A_{1}+v_{3} A_{3}=v_{2} A_{2}=v_{4} A_{4}=0
$$

and

$$
v_{1} B_{1}+v_{3} B_{3}=v_{2} B_{2}=v_{4} B_{4}=0 .
$$

Since $v_{2} \neq 0, A_{2}=B_{2}=0$ holds. This means the equations $\tau_{21}=\rho_{2}^{2} / 2$ and $\rho_{1} \tau_{21}=\rho_{2}^{3} / 6$, which imply $3 \rho_{1}=\rho_{2}$. Hence, we lead to a contradiction by virtue of Lemma 1.7.3.

Case 1.3. $\rho_{1}=\rho_{4}$.

This is equivalent to Case 1.2 .

Case 1.4. $\rho_{2}=\rho_{3}$.

We can assume that $\rho_{1}, \rho_{2}, \rho_{4}$ are distinct. From (E-0), $(\mathrm{E}-1),(\mathrm{E}-21)$, we have the solution

$$
\begin{aligned}
v_{1} & =\frac{-6 \rho_{2} \rho_{4}+2\left(\rho_{2}+\rho_{4}\right)-1}{12\left(\rho_{1}-\rho_{2}\right)\left(\rho_{4}-\rho_{1}\right)} \\
v_{2}+v_{3} & =\frac{-6 \rho_{4} \rho_{1}+2\left(\rho_{4}+\rho_{1}\right)-1}{12\left(\rho_{1}-\rho_{2}\right)\left(\rho_{2}-\rho_{4}\right)}, \\
v_{4} & =\frac{-6 \rho_{1} \rho_{2}+2\left(\rho_{1}+\rho_{2}\right)-1}{12\left(\rho_{2}-\rho_{4}\right)\left(\rho_{4}-\rho_{1}\right)} .
\end{aligned}
$$

Note that each numerator on the right can not vanish by the same reason as in Case 1.2. Then, (1) implies $v_{1} A_{1}=v_{2} A_{2}+v_{3} A_{3}=v_{4} A_{4}=0$. Since $v_{1} \neq 0$, we see $A_{1}=0$, which means $\rho_{1}=0$. Thus, we lead to a contradiction.

Case 1.5. $\rho_{2}=\rho_{4}$ and

Case 1.6. $\rho_{3}=\rho_{4}$. 
Both of them are equivalent to Case 1.4.

Case 2. No two of $\rho_{1}, \rho_{2}, \rho_{3}, \rho_{4}$ are equal.

The equation (1) implies $v_{1} A_{1}=v_{2} A_{2}=v_{3} A_{3}=v_{4} A_{4}=0$. Thus, we distinguish cases according as $v_{i}$ or $A_{i}$ vanishes. We have, however, the following results.

Lemma 1.7.4. The case of $v_{J}=v_{K}=v_{L}=0$ can not occur.

Proof. In this case, $v_{I}=1 / 2$ by (E-0). Then, (E-1) implies $\rho_{I}=1 / 3$. But, they do not satisfy (E-21).

Lemma 1.7.5. The case for $v_{K}=v_{L}=0$ leads to a contradiction.

Proof. The equation $v_{K}=v_{L}=0$ yields a linear system

$$
\begin{aligned}
& v_{I}+v_{J}=\frac{1}{2}, \\
& \rho_{I} v_{I}+\rho_{J} v_{J}=\frac{1}{6}
\end{aligned}
$$

by $(\mathrm{E}-0),(\mathrm{E}-1)$. Since $\rho_{I} \neq \rho_{J}$, this system has the solution

$$
v_{I}=\frac{1-3 \rho_{J}}{6\left(\rho_{I}-\rho_{J}\right)}, \quad v_{J}=\frac{1-3 \rho_{I}}{6\left(\rho_{J}-\rho_{I}\right)} .
$$

Substitution of this into (E-21) and (E-31) implies

$$
\begin{aligned}
& \frac{1}{6}\left(\rho_{I}+\rho_{J}\right)-\frac{1}{2} \rho_{I} \rho_{J}=\frac{1}{12}, \\
& \frac{1}{6}\left(\rho_{I}^{2}+\rho_{I} \rho_{J}+\rho_{J}^{2}\right)-\frac{1}{2} \rho_{I} \rho_{J}\left(\rho_{I}+\rho_{J}\right)=\frac{1}{20} .
\end{aligned}
$$

Put $X=\rho_{I}+\rho_{J}, Y=\rho_{I} \rho_{J}$, we have

$$
\begin{aligned}
& 2 X-6 Y=1, \\
& 10\left(X^{2}-Y\right)-30 X Y=3 .
\end{aligned}
$$

Thus, we easily see that $X=4 / 5, Y=1 / 10$. That is, $\rho_{I}$ and $\rho_{J}$ are equal to the roots of the quadratic equation

$$
x^{2}-\frac{4}{5} x+\frac{1}{10}=0,
$$

which has real distinct roots. On the other hand, we see that

$$
\text { the left on } \begin{aligned}
(E-41) & =v_{I} \rho_{I}^{4}+v_{J} \rho_{J}^{4} \\
& =\frac{1}{6}\left(\rho_{I}+\rho_{J}\right)\left(\rho_{I}^{2}+\rho_{J}^{2}\right)-\frac{1}{2} \rho_{I} \rho_{J}\left(\rho_{I}^{2}+\rho_{I} \rho_{J}+\rho_{J}^{2}\right) \\
& =\frac{19}{600} .
\end{aligned}
$$


which is a contradiction.

Due to the above Lemmas, we distinguish five cases.

Case 2.1. $\quad v_{1}=0$ and $A_{2}=A_{3}=A_{4}=0$. By a similar consideration as in Case 1. we see that $\rho_{2}, \rho_{3}, \rho_{4}$ satisfy

$$
\begin{aligned}
& \rho_{2}+\rho_{3}+\rho_{4}=\frac{9}{7}, \\
& \rho_{2} \rho_{3}+\rho_{3} \rho_{4}+\rho_{4} \rho_{2}=\frac{3}{7}, \\
& \rho_{2} \rho_{3} \rho_{4}=\frac{1}{35} .
\end{aligned}
$$

Hence, they are equal to the distinct roots of the cubic equation (3). Note that, contrary to Case $1, \rho_{1}$ is equal to none of them.

On the other hand, the equation (2) yields $v_{2} B_{2}=v_{3} B_{3}=v_{4} B_{4}=0$. Taking Lemma 1.7.5 into account, we are sufficient to consider the case $B_{2}=B_{3}=B_{4}=0$.

$A_{2}=B_{2}=0$ implies the equations $\tau_{21}=\rho_{2}^{2} / 2$ and $\rho_{1} \tau_{21}=\rho_{2}^{3} / 6$, which give $\rho_{1}=\rho_{2} / 3 . \quad A_{3}=B_{3}=0$ implies a linear system

$$
\begin{aligned}
& \tau_{31}+\tau_{32}=\frac{1}{2} \rho_{3}^{2}, \\
& \rho_{1} \tau_{31}+\rho_{2} \tau_{32}=\frac{1}{6} \rho_{3}^{3},
\end{aligned}
$$

which has the solution

$$
\tau_{31}=\frac{\rho_{3}^{2}\left(3 \rho_{2}-\rho_{3}\right)}{4 \rho_{2}}, \quad \tau_{32}=\frac{\rho_{3}^{2}\left(\rho_{3}-\rho_{2}\right)}{4 \rho_{2}}
$$

Since $\rho_{2}, \rho_{3}, \rho_{4}$ are distinct, (E-0), (E-1), (E-21) give the solution for $v_{2}, v_{3}, v_{4}$ as

$$
\left\{\begin{array}{l}
v_{2}=\frac{-6 \rho_{3} \rho_{4}+2\left(\rho_{3}+\rho_{4}\right)-1}{12\left(\rho_{2}-\rho_{3}\right)\left(\rho_{4}-\rho_{2}\right)} \\
v_{3}=\frac{-6 \rho_{4} \rho_{2}+2\left(\rho_{4}+\rho_{2}\right)-1}{12\left(\rho_{2}-\rho_{3}\right)\left(\rho_{3}-\rho_{4}\right)} \\
v_{4}=\frac{-6 \rho_{2} \rho_{3}+2\left(\rho_{2}+\rho_{3}\right)-1}{12\left(\rho_{3}-\rho_{4}\right)\left(\rho_{4}-\rho_{2}\right)}
\end{array}\right.
$$

(E-44) gives the equation 


$$
\begin{aligned}
v_{4}\left(\rho_{1}^{2} \tau_{41}+\rho_{2}^{2} \tau_{42}+\rho_{3}^{2} \tau_{43}\right) & =\frac{1}{360}-v_{2} \rho_{1}^{2} \tau_{21}-v_{3}\left(\rho_{1}^{2} \tau_{31}+\rho_{2}^{2} \tau_{32}\right) \\
& =\frac{1}{360}-\frac{1}{18} v_{2} \rho_{2}^{4}-v_{3} \rho_{2}^{2}\left(\frac{1}{9} \tau_{31}+\tau_{32}\right) \\
& =\frac{1}{360}-\frac{1}{18} v_{2} \rho_{2}^{4}-\frac{1}{18} v_{3} \rho_{2} \rho_{3}^{2}\left(4 \rho_{3}-3 \rho_{2}\right)
\end{aligned}
$$

by (9). Hence, we may represent the left on (E-54) as the polynomial of $\rho_{2}$ and $\rho_{3}$. By (9) and (10),

$$
\begin{gathered}
v_{2} \rho_{2} \rho_{1}^{2} \tau_{21}+v_{3} \rho_{3}\left(\rho_{1}^{2} \tau_{31}+\rho_{2}^{2} \tau_{32}\right)+v_{4} \rho_{4}\left(\rho_{1}^{2} \tau_{41}+\rho_{2}^{2} \tau_{42}+\rho_{3}^{2} \tau_{43}\right)-\frac{1}{504} \\
=\frac{1}{18} v_{2} \rho_{2}^{5}+\frac{1}{18} v_{3} \rho_{2} \rho_{3}^{3}\left(4 \rho_{3}-3 \rho_{2}\right)+\frac{\rho_{\Delta}}{360}-\frac{1}{18} v_{2} \rho_{2}^{4} \rho_{4} \\
\quad-\frac{1}{18} v_{3} \rho_{2} \rho_{3}^{2} \rho_{4}\left(4 \rho_{3}-3 \rho_{2}\right)-\frac{1}{504} \\
=\frac{1}{37800\left(\rho_{2}-\rho_{3}\right)}\left(525 \rho_{2}^{2} \rho_{3}^{2}-360 \rho_{2}^{2} \rho_{3}-420 \rho_{2} \rho_{3}^{2}\right. \\
\left.-5 \rho_{2}^{2}+260 \rho_{2} \rho_{3}+105 \rho_{3}^{2}-60 \rho_{3}+3\right) .
\end{gathered}
$$

Let us denote the numerator of the above by $\varphi\left(\rho_{2}, \rho_{3}\right)$. The question is whether $\varphi\left(\rho_{2}, \rho_{3}\right)$ vanishes for any pair $\left(\rho_{2}, \rho_{3}\right)$. We have known the values $R_{0}, R_{1}$, $R_{-1}$ which $\rho_{2}$ and $\rho_{3}$ are possible to be equal to. Calculation shows the following:

$$
\begin{aligned}
& \varphi\left(R_{0}, R_{1}\right)=\frac{4}{343}\left(-420 \cos ^{2} \theta-760 \sqrt{3} \sin \theta \cdot \cos \theta-630 \sqrt{2} \cos \theta+60 \sqrt{6} \sin \theta\right. \\
& +189+42 \sqrt{3}) \text {, } \\
& \varphi\left(R_{0}, R_{-1}\right)=\frac{4}{343}\left(-420 \cos ^{2} \theta+760 \sqrt{3} \sin \theta \cdot \cos \theta-630 \sqrt{2} \cos \theta-60 \sqrt{6} \sin \theta\right. \\
& +189-42 \sqrt{3)} \\
& \varphi\left(R_{1}, R_{0}\right)=\frac{4}{343}\left(1350 \cos ^{2} \theta-170 \sqrt{3} \cos \theta \cdot \sin \theta+405 \sqrt{2} \cos \theta-285 \sqrt{6} \sin \theta\right. \\
& -696-42 \sqrt{3)} \\
& \varphi\left(R_{1}, R_{-1}\right)=\frac{4}{343}\left(-930 \cos ^{2} \theta+590 \sqrt{3} \sin \theta \cdot \cos \theta+225 \sqrt{2} \cos \theta-345 \sqrt{6} \sin \theta\right. \\
& +444+42 \sqrt{3}) \\
& \varphi\left(R_{-1}, R_{0}\right)=\frac{4}{343}\left(1350 \cos ^{2} \theta+170 \sqrt{3} \sin \theta \cdot \cos \theta+405 \sqrt{2} \cos \theta+285 \sqrt{6} \sin \theta\right. \\
& -696+42 \sqrt{3}) \\
& \varphi\left(R_{-1}, R_{1}\right)=\frac{4}{343}\left(-930 \cos ^{2} \theta-590 \sqrt{3} \sin \theta \cdot \cos \theta+225 \sqrt{2} \cos \theta+345 \sqrt{6} \sin \theta\right. \\
& +444-42 \sqrt{3}) \text {. }
\end{aligned}
$$


Computation by interval arithmetic shows the following:

$$
\begin{aligned}
& \varphi\left(R_{0}, R_{1}\right) \in[-11.6391,-11.6390], \\
& \varphi\left(R_{0}, R_{-1}\right) \in[-4.70784,-4.70783], \\
& \varphi\left(R_{1}, R_{0}\right) \in[3.20479,3.20480], \\
& \varphi\left(R_{1}, R_{-1}\right) \in[0.821791,0.821792], \\
& \varphi\left(R_{-1}, R_{0}\right) \in[12.1750,12.1751], \\
& \varphi\left(R_{-1}, R_{1}\right) \in[-0.956777,-0.956776] .
\end{aligned}
$$

(On the interval arithmetic, see [8]. Above calculation is carried out by the program made by $\mathrm{K}$. Ichida on HITAC VOS3 at the Educational Center for Information Processing, Kyoto Univ.) None of them vanishes under the condition (4) because every interval given above is away from zero. Thus, we have a contradiction.

Case 2.2. $v_{2}=0$ and $A_{1}=A_{3}=A_{4}=0 . A_{1}=0$ implies $\rho_{1}=0 . \quad$ Then, $\rho_{2}, \rho_{3}, \rho_{4}$ can not vanish. From $(\mathrm{E}-1),(\mathrm{E}-21)$, we have

$$
v_{3}=\frac{1-2 \rho_{4}}{12 \rho_{3}\left(\rho_{3}-\rho_{4}\right)}, \quad v_{4}=\frac{1-2 \rho_{3}}{12 \rho_{4}\left(\rho_{4}-\rho_{3}\right)} .
$$

Substituting these into (E-31), (E-41), we see that

$$
\begin{aligned}
& \rho_{3}+\rho_{4}-2 \rho_{3} \rho_{4}=\frac{3}{5}, \\
& \left(\rho_{3}+\rho_{4}\right)^{2}-2 \rho_{3} \rho_{4}\left(\rho_{3}+\rho_{4}\right)-\rho_{3} \rho_{4}=\frac{2}{5} .
\end{aligned}
$$

Put $X=\rho_{3}+\rho_{4}, Y=\rho_{3} \rho_{4}$, then we have $X=1, Y=1 / 5$. The left on (E-51) is equal to

$$
\begin{gathered}
\frac{1}{12}\left(-2 \rho_{3}^{3} \rho_{4}-2 \rho_{3} \rho_{4}^{3}-2 \rho_{3}^{2} \rho_{4}^{2}+\rho_{3}^{3}+\rho_{3}^{2} \rho_{4}+\rho_{3} \rho_{4}^{2}+\rho_{4}^{3}\right) \\
=\frac{1}{12}\left\{X^{3}-2\left(X^{2}-Y\right) Y-2(X+Y) Y\right\}=\frac{1}{100}
\end{gathered}
$$

which is a contradiction.

Case 2.3. $v_{3}=0$ and $A_{1}=A_{2}=A_{4}=0 . \quad$ Equivalent to Case 2.2.

Case 2.4. $v_{4}=0$ and $A_{1}=A_{2}=A_{3}=0 . \quad$ Equivalent to Case 2.2.

Case 2.5. $A_{1}=A_{2}=A_{3}=A_{4}=0 . A_{1}=0$ implies $\rho_{1}=0$, which means $B_{1}=0$. Then, the equations $\sum v_{i} B_{i}=\sum v_{i} \rho_{i} B_{i}=\sum v_{i} \rho_{i}^{2} B_{i}=0$ yield $v_{2} B_{2}=v_{3} B_{3}$ $=v_{4} B_{4}=0$. Since $v_{2}, v_{3}, v_{4}$ are assumed to be non-zero, we have $B_{2}=B_{3}=B_{4}$ 
$=0 . \quad B_{2}=\rho_{1} \tau_{21}-\rho_{2}^{3} / 6=0$ implies $\rho_{2}=0$ because $\rho_{1}=0$. This contradicts the assumption that no two of $\rho_{i}$ are equal.

Now, we have accomplished to investigate the whole cases. In conclusion, we have

Theorem 8. The explicit (1,4)-stage formula can not attain order 7 . Its attainable order is 6.

Note. Shintani gives $(1,4)$-stage formula with parameters $v_{1}=1 / 20, v_{2}$ $=7(7+\sqrt{21}) / 360, \quad v_{3}=8 / 45, \quad v_{4}=7(7-\sqrt{21}) / 360, \quad \rho_{1}=0 . \quad \rho_{2}=\left(7-v^{\sqrt{21}}\right) / 14, \quad \tau_{21}$ $=(5-\sqrt{21}) / 28, \rho_{3}=1 / 2, \tau_{31}=(3-\sqrt{21}) / 192, \tau_{32}=(21+\sqrt{21}) / 192, \rho_{4}=(7+\sqrt{21}) /$ $14, \tau_{41}=(21+5 \sqrt{21}) / 294, \quad \tau_{42}=(\sqrt{21}-3) / 84, \quad \tau_{43}=(21+\sqrt{21}) / 147$. These parameters are also not unique solution of $(E-0)-(E-46)$.

\section{References}

[1] Butcher, J. C., Coefficients for the study of Runge-Kutta integration processes, $J$. Austral. Math. Soc., 3 (1963), 185-201.

[2] _- , On Runge-Kutta processes of high order, J. Austral. Math. Soc., 4 (1964), 179-194.

[ 3 ] - -, Implicit Runge-Kutta processes, Math. Comput., 18 (1964), 50-64.

[4] - On the attainable order of Runge-Kutta methods, Math. Comput., 19 (1965), 408-417.

[ 5 ] Cash, J. R., High order methods for the numerical integration of ordinary differential equations, Numer. Math., 30 (1978), 385-409.

[6] Hearn, A. C., REDUCE 2 User's Manual, second ed., Univ. Utah, March 1973.

[7] Kaps, P. and Rentrop. P., Generalized Runge-Kutta methods of order four with stepsize control for stiff ordinary differential equations, Numer. Math., 33 (1979), 55-68.

[8] Moore, R. E., Interval Analysis, Prentice-Hall Inc., New Jersey, 1966.

[9] Nørsett, S. P. and Walfbrandt, A., Order conditions for Rosenbrook-type method, Numer. Math., 32 (1979), 1-15.

[10] Riordan, J., Derivatives of composite functions, Bull. A. M. S., 52 (1946), 664-667.

[11] Rosenbrock, H. H., Some general implicit processes for the numerical solution of differential equations, Computer J., 5 (1963), 329-330.

[12] Shintani, H., On one-step methods utilizing the second derivative, Hiroshima Math. J., 1 (1971), 349-372.

[13] - On explicit one-step methods utilizing the second derivative, Hiroshima Math. J., 2 (1972), 353-368.

[14] Toda, H., On the truncation error of a limiting formula of Runge-Kutta methods, Res. Electrotech. Lab., No. 772, 1977.

[15] Urabe, M., An implicit one-step method of high order accuracy for the numerical integration of ordinary differential equations, Numer. Math., 15 (1970), 151-164. 